\title{
Distributed Event-Triggered Output Synchronization of Complex-Valued Memristive Reaction-Diffusion Complex Networks with Spatial Sampled-Data
}

\author{
Tiane Chen (iD) and Zaihe Cheng \\ School of Internet of Things, Wuxi Institute of Technology, Wuxi 214121, China \\ Correspondence should be addressed to Tiane Chen; chente@wxit.edu.cn
}

Received 4 February 2021; Revised 24 February 2021; Accepted 13 March 2021; Published 26 March 2021

Academic Editor: Xue-bo Jin

Copyright (C) 2021 Tiane Chen and Zaihe Cheng. This is an open access article distributed under the Creative Commons Attribution License, which permits unrestricted use, distribution, and reproduction in any medium, provided the original work is properly cited.

\begin{abstract}
This study addresses the problem of output quasisynchronization for coupled complex-valued memristive reaction-diffusion complex networks via the distributed event-triggered control scheme. First, by using the separate method, set value mapping, and intermediate value theorem, the complex-valued memristive reaction-diffusion complex networks can be transferred into two semi-uncertain real-valued reaction-diffusion complex networks. Second, a distributed output piecewise event-triggered control (OPETC) scheme with spatial sampled-data is first proposed including a spatial sampling event-triggered generator and spatiotemporal sampling state feedback controller. Furthermore, this scheme can effectively save the measurement resources and lower the update rate of controllers in spatial and time domain. Third, the synchronization analysis is considered by utilizing an appropriate Lyapunov function, the Halanay inequality, and the improved Wirtinger inequality. Subsequently, several output event-triggered quasisynchronization criteria are derived. The relations among event trigger conditions, spatial sampling interval, convergence rate, and control gain are given by rigorous mathematical derivation. Finally, multiple simulations are compared to substantiate the validation of the OPETC scheme.
\end{abstract}

\section{Introduction}

Complex networks have excited continuous interest for their nonlinear learning characteristics and have been extensively implemented in various domains such as deep learning [1-3], natural language analyzation, optimization computing, and image segments. When two or more networks interact, coupled complex networks, such as coupled neural networks (CNNs), can be formed and have attracted much considerable attentions for their significant collective dynamical behaviour. As an important collective dynamical behaviour, synchronization means that the states of complex networks are always consistent over time. Many crucial results about the synchronization of $\mathrm{CNNs}$ have been achieved in literatures [4-7]. For instance, Bai and $\mathrm{Xu}$ [4] studied the synchronization of CNNs with hybrid coupling, Luo and Yao [5] investigated the finite-time synchronization of uncertain complex dynamic networks, Wang et al. [6] researched the synchronization of coupled CNNs via the pinning method, and Ding et al. [7] considered the impulsive synchronization of complex networks.

It should be highlighted that most research studies suppose that the electromagnetic fields of complex networks are uniform, and the networks states vary only with time. In fact, inhomogeneous electromagnetic fields inevitably exist in cellular networks [8], genetic regulatory networks, and traffic networks. In nonuniform electromagnetic fields, the networks states in different spatial positions are distinct and change simultaneously. This phenomenon can be expressed as the reaction-diffusion (RD) term in mathematical models. Then, the CNNs model is transformed into coupled RD neural networks (CRDNNs) with infinite dimensional states. Up to now, plenty of impressive studies on the synchronization of CRDNNs have been achieved. The authors 
investigated the impulsive synchronization [9], the adaptive synchronization [10], and the passivity [11] of CRDNNs. The abovementioned works assumed that the global spatial state information was known, and all these research studies aimed to synchronize all states. In some applications, it is costly and not necessary to measure the whole infinite dimensional state and to synchronize all states. Therefore, it is valuable to research partial state synchronization using incomplete measurements. Partial state synchronization named output synchronization (OS) has been investigated in literatures [12-15]. The authors [16] studied OS of CRDNNs with input constraints via continuous time control, and the authors [17] investigated OS of CRDNNs with spatial sampled-data via continuous time control. To our knowledge, few results about OS of CRDNNs have been investigated via noncontinuous time control. This is our first motivation for this study.

Abovementioned investigations of complex networks have intensively emerged with state-independent weights realized by resistors. As a special resistor, memristor has been proposed by Chua [18] and has been realized first by HP Labs. Memristor can retain its history state owing to its switch characteristic which makes the memristor an attractive candidate to emulate the biological brain to improve machine intelligence $[19,20]$. Consequently, memristive reaction-diffusion neural networks (MRDNNs) can be constructed with richer properties than common CRDNNs. Accordingly, it is significant to study MRDNNs with statedependent weight. Until now, many worthwhile results about MRDNNs have been achieved. Global synchronization [21, 22], exponential Lag synchronization [23], and fixed-time synchronization [24] of MRDNNs have been studied. Nonetheless, the mismatch of the master-slave system due to state-dependent parameters inevitably causes the synchronization error of the collective behaviour to change in a certain range. That is quasisynchronization [25]. Actually, the research results on quasisynchronization of MRDNNs have not yet been published. This is our second motivation for this study.

Different from real-valued neural networks, complexvalued neural networks own more complicated properties. Some scientific questions, such as the phase-sensitive detection [26] and exclusive or problem [27] in optical signal processing, can only be addressed by complex-valued neural networks. Complex-valued MRDNNs (CMRDNNs) have complex-valued states, state-dependent parameter, and complex-valued activation function which are more general than real-valued MRDNNs. Hence, it is necessary to investigate CMRDNNs. Besides, the existing difficulty is that complex-valued functions may not be bounded or analytic [28]. Many assumptions about real-valued functions cannot be utilized in the complexed-valued domain without any preprocessing. Thus, the general approach is to convert complex-valued functions into two parts: real and imaginary parts. Using this conversion method, intermittent control synchronization [28], exponential sampled-data synchronization, [29] and pinning control synchronization [30] of complex-valued memristive networks were investigated without considering $\mathrm{RD}$ term. Nevertheless, the synchronization problem of CMRDNNs has rarely been addressed. Song et al. [31] studied the finite-time synchronization of CMRDNNs with known full state information and activation function bounds. Therefore, it is meaningful to further research the synchronization problem of CMRDNNs with incomplete measurements.

To achieve the synchronization of complex networks, many control strategies have been studied, such as continuous time control [16], sampled-data estimation and control [29, 32], and intermittent control [28]. Among these control strategies, sampled-data control has received wider attention because this method can reduce communication bandwidth. Sampled-data control includes time-triggered control and event-triggered control [33-35]. Compared with the time-triggered control mechanism, the event-triggered control scheme updates information only when the measurement error satisfies the triggering threshold condition, which leads to a lower information exchange rate. Therefore, the event-triggered sampling control strategy performs better than the time-triggered method [36]. Event trigger's form can be considered as $\|e(x, t)\|^{2}>\gamma\|z(x, t)\|^{2}+\eta(t)$. The event trigger is considered static one when the function $\eta(t)$ is a constant, and the event trigger is considered dynamic one when the derivative of the function $\eta(t)$ is not zero [37]. Thus, the event-triggered control strategy can be classified into dynamical event triggers and static event triggers according to the function $\eta(t)$. Meanwhile, the event-triggered control can be classified into centralized control and distributed control according to the scope of the controller. In large-scale coupled networks, it is difficult to realize complete centralized control. Thus, further research on distributed event-triggered control is crucial, and one of the primary problems to be prevented by event-triggered control scheme is Zeno phenomenon. As far as we know, the distributed event-triggered control strategy for synchronization of CMRDNNs with incomplete spatial measurement has not been studied so far. This is our third motivation for this study.

Motivated by the abovementioned analysis, the main aim of this work is to establish the output quasisynchronization conditions of CMRDNNs via distributed event-triggered partial spatial control strategy. More specially, some questions should be solved: How to obtain the error model of the masterslave networks systems with state-dependent parameters? How to design an efficient distributed piecewise event-triggered control strategy using incomplete measurement to achieve output synchronization? What are the effects of dynamic event trigger and static event trigger on quasisynchronization? To achieve the control goal and answer the above three questions, there are three innovations in this study:

(1) A general CMRDNNs mathematical model with the output matrix is proposed. By using set-valued mappings, differential inclusions, and nonfragile techniques, the real-valued synchronization error model with uncertainty parameters is derived, and the assumption conditions about activation functions are more relaxed.

(2) We establish a distributed output piecewise eventtriggered control (OPETC) strategy with spatial 
sampled-data to ensure the convergent speed and to reduce the communication cost. By using some inequalities and an appropriate Lyapunov functional, several universal criteria guaranteeing the output quasisynchronization of the discussed networks are established, where the relations among diffusion coefficients, control gains, memristive complexvalued range, and time delays are clear.

(3) By comparing the simulation results of time trigger, dynamical event trigger, static event trigger, and piecewise event trigger, the high efficiency of the OPETC strategy is verified.

The structure of this study is as follows: the mathematical model is established and some assumptions are given. Then, the real-valued synchronization error models with uncertainty parameters are deduced in Section 2. A distributed piecewise event-triggered control strategy with spatial sampled-data is designed, and several universal criteria guaranteeing the output quasisynchronization of the discussed networks are deduced in Section 3. Simulation examples are given to compare the effect of different triggers and to corroborate the quasisynchronization conditions in Section 4. Section 5 draws the conclusion of this study and clarifies our future research.

\section{Preliminaries}

In this section, we provide some necessary lemmas and show the process of deriving the mathematical model of the masterslave networks system to two real-valued error models.

2.1. Notations. Let $\mathcal{N}=\{0,1,2, \ldots\}$ and $\mathscr{N}^{+}=\{1,2, \ldots\}$. For each $n \in \mathcal{N}^{+}, \mathcal{N}_{n}=\{0,1,2, \ldots, n\}$ and $\mathscr{N}_{n}^{+}=\{1,2, \ldots, n\} . \mathscr{R}$ and $\mathscr{C}$ denote the set of real-valued space and complexvalued space, respectively. Similarly, $\mathscr{R}^{n}$ and $\mathscr{C}^{n}$ represent the sets of $n$-dimensional real-valued and $n$-dimensional complex-valued vectors. $\mathscr{R}^{n \times N}$ and $\mathscr{C}^{n \times N}$ describe the sets of $n \times N$ real-valued matrices and complex-valued matrices. The Hilbert space of square integrable functions over $\Omega$ is denoted by $\mathscr{L}_{n}^{2}(\Omega)$ with norm $\|z(x, \cdot)\|^{2}=\int_{\Omega} z^{T}(x, \cdot) z$ $(x, \cdot) \mathrm{d} x, \Omega=(0, l)$, and $n \in \mathscr{N}^{+} . \mathscr{I}_{n}$ is a $n$-dimensional identity matrix. $\mathscr{H}_{n}^{1}(\Omega)=\left\{z \in \mathscr{L}_{n}^{2}(\Omega) \mid / \mathrm{d} z \mathrm{~d} x \in \mathscr{L}_{n}^{2}(\Omega)\right\}$ is a Sobolev space which is also a subspace of $\mathscr{L}_{n}^{2}(\Omega)$. T denotes the matrix transposition, and $\otimes$ represents the Kronecker product. $l$ denotes the imaginary part with $\iota=\sqrt{-1}$. $z(x, \cdot)=z^{R}(x, \cdot)+\imath z^{I}(x, \cdot), z^{R}(x, \cdot) \in \mathscr{R}$, and $z^{I}(x, \cdot) \in \mathscr{R}$.

2.2. Model Transform. In a master-slave system scheme, there are two coupled networks with the same structure and diverse initial values. The master-coupled complex-valued memristive reaction-diffusion networks can be expressed as

$$
\left\{\begin{array}{l}
\frac{\partial \widetilde{z}_{i}(x, t)}{\partial t}=-A \widetilde{z}_{i}(x, t)+B \frac{\partial^{2} \widetilde{z}_{i}(x, t)}{\partial x^{2}}+c\left(\widetilde{z}_{i}(x, t)\right) f_{i}\left(\widetilde{z}_{i}(x, t)\right)+\sum_{j=1}^{N} d_{i j} \Gamma g_{j}\left(\widetilde{z}_{j}\left(x, t-\tau_{j}(t)\right)\right) \\
\tilde{y}_{i}(x, t)=\beta \widetilde{z}_{i}(x, t)
\end{array}\right.
$$

where $\widetilde{z}_{i}(x, t)=\left(\widetilde{z}_{i 1}(x, t), \cdots, \widetilde{z}_{\text {in }}(x, t)\right)^{T} \in \mathscr{H}_{n}^{1}(\Omega)$ denotes the state information of the $i^{\text {th }}$ networks with $i \in \mathcal{N}_{N}^{+}$, $t \in\left[t_{0},+\infty\right)$ and $t_{0} \geq 0 ; x \in \Omega$ corresponds to the space variable; $A \in \mathscr{R}^{n \times n}$ represents the self-inhibition coefficient; $B \in \mathscr{R}^{n \times n}$ denotes the diffusion coefficient; $\tau_{j}(t)$ represents the nonnegative time-varying transmission delay from the $j^{\text {th }}$ networks to the $i^{\text {th }}$ networks; $\bar{\tau}=\max \left(\tau_{j}(t)\right) ; c\left(\widetilde{z}_{\mathrm{i}}(x, t)\right)$ is the connection weights with memristive characteristic; $f_{i}(\cdot)$ corresponds to the neural activation vector function of the $i^{\text {th }}$ networks; $\Gamma \in \mathscr{R}^{n \times n}$ is the inner coupling matrix; $D=$ $\left(d_{i j}\right)_{N \times N} \in \mathscr{C}^{N \times N}$ denotes the coupling strength matrix where $d_{i i}=-\sum_{j=1, j \neq i}^{N} d_{i j} ; g_{j}(\cdot)$ denotes the complex-valued state function; $\widetilde{y}_{i}(x, t) \in \mathscr{C}^{\mathrm{r} \times 1}$ is the output vector; $\beta \in \mathscr{R}^{r \times n}$ represents the output matrix; and $\beta^{\mathrm{T}} \beta$ is the idempotent matrix.

The initial values and the Dirichlet boundary values of the master networks are given by

$$
\begin{aligned}
& \widehat{z}_{i}(x, s)=\widehat{v}_{i}(x, s),(x, t) \in \Omega \times\left[t_{0}-\bar{\tau}, t_{0}\right], \\
& \widehat{z}_{i}(0, t)=\widehat{z}_{i}(l, t)=0,(x, t) \in \partial \Omega \times\left[t_{0}-\bar{\tau},+\infty\right],
\end{aligned}
$$

,where $\widetilde{v}_{i}(x, s)$ is a complex-valued function.

Similarly, the slave networks can be designed as

$$
\left\{\begin{array}{l}
\frac{\partial \widehat{z}_{i}(x, t)}{\partial t}=-A \widehat{z}_{i}(x, t)+B \frac{\partial^{2} \widehat{z}_{i}(x, t)}{\partial x^{2}}+c_{i}\left(\widehat{z}_{i}(x, t)\right) f_{i}\left(\widehat{z}_{i}(x, t)\right)+\sum_{j=1}^{N} d_{i j} \Gamma g_{j}\left(\widehat{z}_{j}\left(x, t-\tau_{j}(t)\right)\right)+\widehat{u}_{i}(x, t), \\
\widehat{y}_{i}(x, t)=\beta \widehat{z}_{i}(x, t),
\end{array}\right.
$$


where $\widehat{u}_{i}(x, t) \in \mathscr{H}_{n}^{1}(\Omega)$ is the control input of the $i^{\text {th }}$ networks to achieve output synchronization; the initial condition and the Dirichlet boundary condition of the slave networks are given by

$$
\begin{aligned}
& \widehat{z}_{i}(x, s)=\widehat{v}_{i}(x, s),(x, t) \in \Omega \times\left[t_{0}-\bar{\tau}, t_{0}\right], \\
& \widehat{z}_{i}(0, t)=\widehat{z}_{i}(l, t)=0,(x, t) \in \partial \Omega \times\left[t_{0}-\bar{\tau},+\infty\right],
\end{aligned}
$$

where $\widehat{v}_{i}(x, s)$ is a complex-valued function.

Suppose that $c_{i}^{0}$ and $c_{i}^{1}$ are two complex-valued constants and $v_{i}(x, t) \in \mathscr{C}$. According to the switch property of memristor, the connection weight $c_{i}\left(v_{i}(x, t)\right)$ can be defined as

$$
c_{i}\left(v_{i}(x, t)\right)= \begin{cases}c_{i}^{0}, & v_{i}(\cdot, t) \uparrow, \\ c_{i}^{1}, & v_{i}(\cdot, t) \downarrow, \\ \lim _{s \longrightarrow t^{-}} c_{i}\left(v_{i}(\cdot, t)\right), & v_{i}(\cdot, t) \text { unchange, }\end{cases}
$$

where $\quad c_{i}^{0}=c_{i}^{0 R}+\iota c_{i}^{0 I}, \quad c_{i}^{1}=c_{i}^{1 R}+\iota c_{i}^{1 I}, \quad \bar{c}_{i}^{R}=\max \left\{c_{i}^{0 R}, c_{i}^{1 R}\right\}$, $\underline{c}_{i}^{R}=\min \left\{c_{i}^{0 R}, c_{i}^{1 R}\right\}, \bar{c}_{i}^{I}=\max \left\{c_{i}^{0 I}, c_{i}^{1 I}\right\}$, and $\underline{c}_{i}^{I}=\min \left\{c_{i}^{0 I}, c_{i}^{1 I}\right\}$.

We can formulate equations (1) and (3) in a compact matrix form as

$$
\left\{\begin{array}{l}
\frac{\partial \widetilde{z}(x, t)}{\partial t}=-\left(I_{N} \otimes A\right) \widetilde{z}(x, t)+\left(I_{N} \otimes B\right) \frac{\partial^{2} \widetilde{z}(x, t)}{\partial x^{2}}+C(\widetilde{z}(x, t)) f(\widetilde{z}(x, t))+D \otimes \Gamma g(\widetilde{z}(x, t-\tau(t))) \\
\widetilde{y}(x, t)=\left(I_{N} \otimes \beta\right) \widetilde{z}(x, t), \\
\frac{\partial \widehat{z}(x, t)}{\partial t}=-\left(I_{N} \otimes A\right) \widehat{z}(x, t)+\left(I_{N} \otimes B\right) \frac{\partial^{2} \widehat{z}(x, t)}{\partial x^{2}}+C(\widehat{z}(x, t)) f(\widehat{z}(x, t))+D \otimes \Gamma g(\widehat{z}(x, t-\tau(t)))+u(x, t), \\
\hat{y}(x, t)=\left(I_{N} \otimes \beta\right) \widehat{z}(x, t),
\end{array}\right.
$$

where $\quad \tilde{z}(x, t)=\left(\widetilde{z}_{1}^{T}(x, t), \ldots, \widetilde{z}_{N}^{T}(x, t)\right)^{T}, \quad \widehat{z}(x, t)=\left(\widehat{z}_{1}^{T}\right.$ $\left.(x, t), \ldots, \widehat{z}_{N}^{T}(x, t)\right)^{T}, u(x, t)=\left[u_{1}^{T}(x, t), u_{2}^{T}(x, t), \ldots, u_{N}^{T}\right.$ $(x, t)]^{T}, C(\widetilde{z}(x, t))=\left(c_{1}\left(\widetilde{z}_{1}^{T}(x, t)\right), \ldots, c_{N}\left(\widetilde{z}_{N}^{T}(x, t)\right)\right)^{T}, C$ $(\widehat{z}(x, t))=\left(c_{1}\left(\widehat{z}_{1}^{T}(x, t)\right), \ldots, c_{N}\left(\widehat{z}_{N}^{T}(x, t)\right)\right)^{T}, \quad f(\cdot)=\left(f_{1}^{T}\right.$ $\left.(\cdot), \ldots, f_{N}^{T}(\cdot)\right)^{T}, g(\cdot)=\left(g_{1}^{T}(\cdot), \ldots, g_{N}^{T}(\cdot)\right)^{T}, \tilde{y}(x, t)=\left(\widetilde{y}_{1}^{T}\right.$ $\left.(x, t), \ldots, \tilde{y}_{N}^{T}(x, t)\right)^{T}, \quad$ and $\quad \hat{y}(x, t)=\left(\hat{y}_{1}^{T}(x, t) \ldots, \quad \hat{y}_{N}^{T}\right.$ $(x, t))^{T}$.

Assume that the master-slave networks (equations (1) and (3)) with Dirichlet boundary conditions have unique continuous solutions $\widetilde{z}_{i}(x, t)$ and $\widehat{z}(x, t)$, respectively.

Remark 1. The authors [17] considered the reaction-diffusion term and output matrix. The mathematical model in [38] included memristive parameters and reaction-diffusion terms without complex-valued state and output matrix. The authors [31] investigated complex-valued states, memristive parameters, and reaction-diffusion term without considering output matrix. Different from literatures $[17,31,38]$, this study introduces complex value states, memristive parameters, reaction-diffusion terms, and output matrices into the mathematical model, which are more general and practical in applications.

Our goal is to synchronize the master system (equation (1)) and the slave system (equation (3)). Then, we naturally need to derivate the error model of the master-slave system. However, many assumptions about real-valued functions cannot be utilized in the complexed-valued domain. Meanwhile, the existence of memristive parameters leads to parameter mismatch of the master networks system and the slave one. Obviously, there are some difficulties in deriving the error model directly. Thus, the model should be converted. First, the complex-valued model is converted into two real-valued models by using some assumptions. Second, the real-valued synchronization error model can be obtained by using different techniques.

Assumption 1. $z(x, t), C(z(x, t))$, and $f(z(x, t))$ can be divided into real and imaginary parts, respectively.

$$
\left\{\begin{array}{l}
z(x, t)=z^{R}(x, t)+\iota z^{I}(x, t), \\
C(z(x, t))=C^{R}(z(x, t))+\iota C^{I}(z(x, t)), \\
f(z(x, t))=f^{R}(z(x, t))+\iota f^{I}(z(x, t)),
\end{array}\right.
$$

where $z^{R}(x, t), C^{R}(z(x, t))$, and $f^{R}(z(x, t))$ are the real parts; $z^{I}(x, t), C^{I}(z(x, t))$, and $f^{I}(z(x, t))$ are the imaginary parts.

Lemma 1 see ([39]). For any function $\{v(\xi) \mid \xi \in[a, b]$, $\left.v(\xi) \in \mathscr{H}_{n}^{1}(a, b)\right\}$. If $x(a)=0$ or $x(b)=0$, then

$$
\int_{a}^{b} v^{T}(\xi) v(\xi) \mathrm{d} \xi \leq \frac{4(b-a)^{2}}{\pi^{2}} \int_{a}^{b}\left(\frac{\partial v(\xi)}{\partial \xi}\right)^{T} \frac{\partial v(\xi)}{\partial \xi} \mathrm{d} \xi
$$

Corollary 1 see $([40,41])$. For any function $\{x(\xi) \mid \xi \in$ $\left.[a, b], x(\xi) \in \mathscr{H}_{n}^{1}(a, b)\right\}, c=a$ or $b$. If $r_{0}, r_{1}, r_{2}, r_{3}>0$, then the following integral inequalities hold, 


$$
-r_{0} \int_{a}^{b}\left(\frac{\partial x(\xi)}{\partial \xi}\right)^{2} \mathrm{~d} \xi-r_{1} \int_{a}^{b} x^{2}(c) \mathrm{d} \xi \leq-r_{2} \int_{a}^{b} x^{2}(\xi) \mathrm{d} \xi
$$

where $r_{2}=\pi^{2} r_{0} r_{1} /\left(\pi^{2} r_{0}+4(b-a)^{2} r_{1}\right)$.

Similarly, we can get

$$
-r_{0} \int_{a}^{b}\left(\frac{\partial x(\xi)}{\partial \xi}\right)^{2} \mathrm{~d} \xi+r_{1} x^{2}(c) \leq r_{3} \int_{a}^{b} x^{2}(\xi) \mathrm{d} \xi
$$

where $r_{3}=\pi^{2} r_{0} r_{1} /\left(\pi^{2} r_{0}-4(b-a)^{2} r_{1}\right)$.

Lemma 2 see $([25,42])$. Assume that the function $W(t) \geq 0$ for all $t \in(-\infty,+\infty)$,

$$
\begin{cases}\frac{\mathrm{d} W(t)}{\mathrm{d} t} \leq \alpha(t) W(t)+\beta(t) \sup _{t-\tau(t) \leq s \leq t} W(s)+\gamma(t), & t \geq t_{0} \\ W(t)=|\psi(t)|, & t \leq t_{0}\end{cases}
$$

where $\psi(t)$ is bounded and continuous and three functions $-\alpha(t), \beta(t)$, and $\gamma(t)$ are continuous and positive. If there exists $\sigma>0$ and $\alpha(t)+\beta(t) \leq-\sigma$ for $t \geq t_{0}$, then we have $W(t) \leq(\bar{\gamma} / \sigma)+\sup _{t-\tau(t) \leq s \leq t} W(s) \exp \left(-\underline{\eta}\left(t-t_{0}\right)\right)$, where $\bar{\gamma}=$ $\sup _{t_{0} \leq t} \gamma(t)$ and $\underline{\eta}=\inf _{t_{0} \leq t}\{\eta(t): \bar{\eta}(t)+\alpha(t)+\beta(t) \exp$ $(\eta(t) \tau(t))=0\}$.

Under Assumption 1, equation (6) can be divided into two real-valued mathematical models as

$$
\begin{aligned}
& \left\{\begin{array}{l}
\frac{\partial \widetilde{z}^{R}(x, t)}{\partial t}=-\left(I_{N} \otimes A\right) \widetilde{z}^{R}(x, t)+\left(I_{N} \otimes B\right) \frac{\partial^{2} \widetilde{z}^{R}(x, t)}{\partial x^{2}}+C^{R}(\widetilde{z}(x, t)) f^{R}(\widetilde{z}(x, t)), \\
-C^{I}(\widetilde{z}(x, t)) f^{I}(\widetilde{z}(x, t))+D \otimes \Gamma g^{R}(\widetilde{z}(x, t-\tau(t))), \\
\tilde{y}^{R}(x, t)=\left(I_{N} \otimes \beta\right) \tilde{z}^{R}(x, t), \\
\frac{\partial \widetilde{z}^{I}(x, t)}{\partial t}=-\left(I_{N} \otimes A\right) \widetilde{z}^{I}(x, t)+\left(I_{N} \otimes B\right) \frac{\partial^{2} \tilde{z}^{I}(x, t)}{\partial x^{2}}+C^{R}(\widetilde{z}(x, t)) f^{I}(\widetilde{z}(x, t)), \\
+C^{I}(\tilde{z}(x, t)) f^{R}(\tilde{z}(x, t))+D \otimes \Gamma g^{I}(\tilde{z}(x, t-\tau(t))), \\
\tilde{y}^{I}(x, t)=\left(I_{N} \otimes \beta\right) \tilde{z}^{I}(x, t),
\end{array}\right. \\
& \left\{\begin{array}{l}
\frac{\partial \widehat{z}^{R}(x, t)}{\partial t}=-\left(I_{N} \otimes A\right) \widehat{z}^{R}(x, t)+\left(I_{N} \otimes B\right) \frac{\partial^{2} \widehat{z}^{R}(x, t)}{\partial x^{2}}+C^{R}(\widehat{z}(x, t)) f^{R}(\widehat{z}(x, t)), \\
-C^{I}(\widehat{z}(x, t)) f^{I}(\widehat{z}(x, t))+D \otimes \Gamma g^{R}(\widehat{z}(x, t-\tau(t)))+u^{R}(x, t), \\
\widehat{y}^{R}(x, t)=\left(I_{N} \otimes \beta\right) \widehat{z}^{R}(x, t), \\
\frac{\partial \widehat{z}^{I}(x, t)}{\partial t}=-\left(I_{N} \otimes A\right) \widehat{z}^{I}(x, t)+\left(I_{N} \otimes B\right) \frac{\partial^{2} \widehat{z}^{I}(x, t)}{\partial x^{2}}+C^{R}(\widehat{z}(x, t)) f^{I}(\widehat{z}(x, t)), \\
+C^{I}(\widehat{z}(x, t)) f^{R}(\widehat{z}(x, t))+D \otimes \Gamma g^{I}(\widehat{z}(x, t-\tau(t)))+u^{I}(x, t), \\
\hat{y}^{I}(x, t)=\left(I_{N} \otimes \beta\right) \widehat{z}^{I}(x, t) .
\end{array}\right.
\end{aligned}
$$


Let $\underline{C}_{i}=\operatorname{diag}\left\{\underline{c}_{1}, \ldots, \underline{c}_{n}\right\}, \quad \bar{C}_{i}=\operatorname{diag}\left\{\bar{c}_{1}, \ldots, \bar{c}_{n}\right\}, \quad$ valued mapping [43], equations (12) and (13) can be $\underline{C}=\operatorname{diag}\left\{\underline{C}_{i}\right\}_{N \times N} \in \mathscr{C}^{n N \times n N}, \quad \bar{C}=\operatorname{diag}\left\{\bar{C}_{i}\right\}_{N \times N} \in \mathscr{C}^{n N \times n N}$, expressed as the differential inclusions: and $C(v(x, t)) \in \operatorname{co}\{\underline{C}, \bar{C}\}$. Using some properties of set-

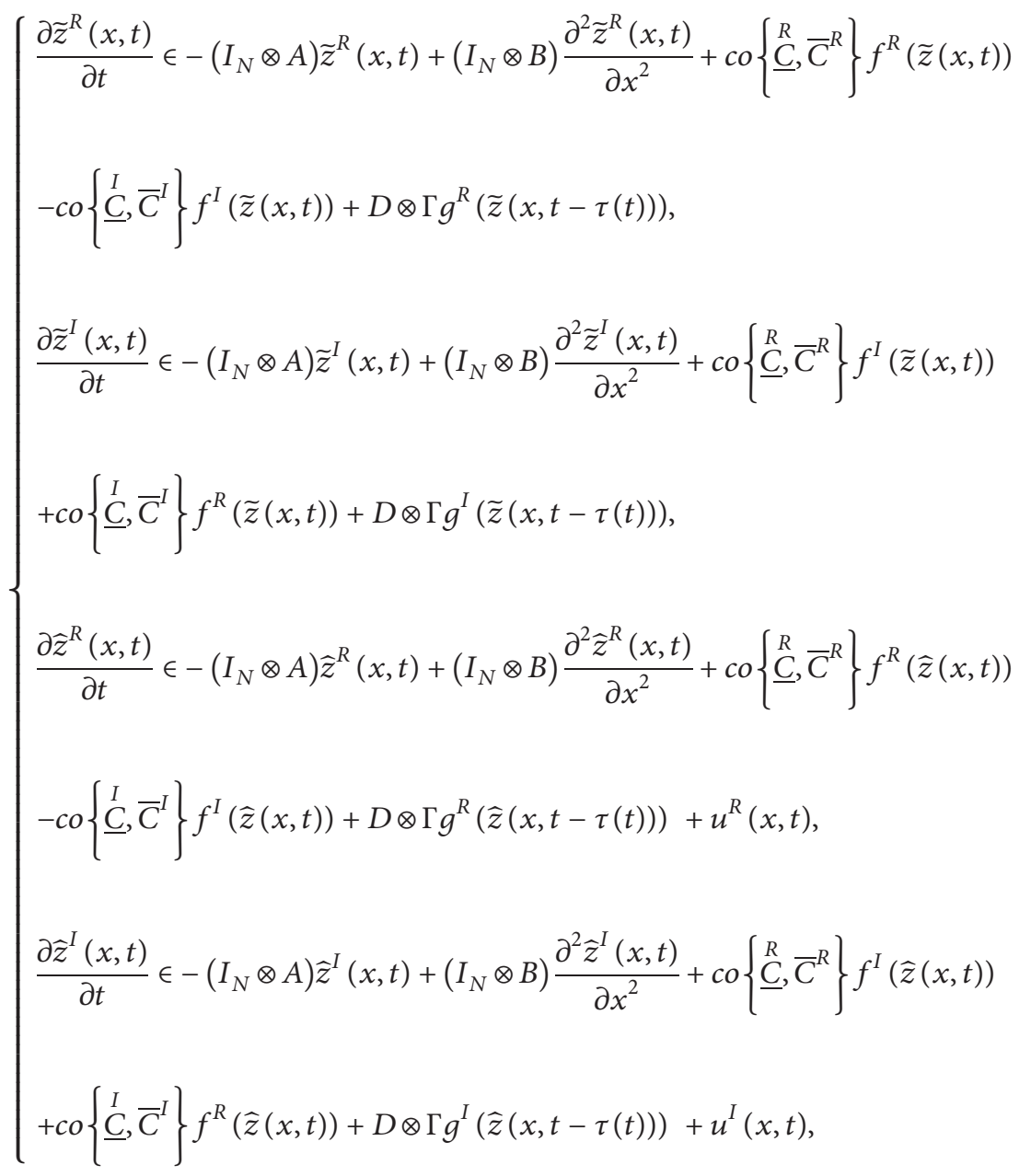

where $\underline{C}_{i}^{R}=\operatorname{diag}\left\{\underline{c}_{1}^{R}, \ldots, \underline{c}_{n}^{R}\right\}, \bar{C}_{i}^{R}=\operatorname{diag}\left\{\bar{c}_{1}^{R}, \ldots, \bar{c}_{n}^{R}\right\}, \quad \underline{C}_{i}^{I}=$ $\operatorname{diag}\left\{\underline{c}_{1}^{I}, \ldots, \underline{c}_{n}^{I}\right\}, \bar{C}_{i}^{I}=\operatorname{diag}\left\{\bar{c}_{1}^{I}, \ldots, \bar{c}_{n}^{I}\right\}, \quad \underline{C}^{R}=\operatorname{diag}\left\{\underline{C}_{i}^{R}\right\}_{N \times N}$, $\bar{C}^{R}=\operatorname{diag}\left\{\bar{C}_{i}^{R}\right\}_{N \times N}, \underline{C}^{I}=\operatorname{diag}\left\{\underline{C}_{i}^{I}\right\}_{N \times N}, \bar{C}^{I}=\operatorname{diag}\left\{\bar{C}_{i}^{I}\right\}_{N \times N}$, $\underline{C}^{R}, \bar{C}^{R}, \underline{C}^{I}, \bar{C}^{I} \in \mathscr{C}^{n N \times n N}, C^{R}(\widetilde{z}(x, t)) \in \operatorname{co}\left\{\underline{C}^{R}, \bar{C}^{R}\right\}$, and $C^{I}$ $(\widetilde{z}(x, t)) \in \operatorname{co}\left\{\underline{C}^{I}, \bar{C}^{I}\right\}$.

Assumption 2. $f^{R}(\cdot), f^{I}(\cdot), g^{R}(\cdot)$, and $g^{I}(\cdot)$ are the bounded activation functions and are Lipschitz continuous. There exist positive values $h_{f}^{R}, h_{f}^{I}, h_{g}^{R}, h_{g}^{I}$ as

$$
\left\{\begin{array}{l}
\left\|f^{R}\left(\xi_{1}\right)-f^{R}\left(\xi_{2}\right)\right\|^{2} \leq h_{f}^{R}\left\|\xi_{1}-\xi_{2}\right\|^{2}, \\
\left\|f^{I}\left(\xi_{1}\right)-f^{I}(\widetilde{z}(x, t))\right\|^{2} \leq h_{f}^{I}\left\|\xi_{1}-\xi_{2}\right\|^{2}, \\
\left\|g^{R}\left(\xi_{1}\right)-g^{R}\left(\xi_{2}\right)\right\|^{2} \leq h_{g}^{R}\left\|\xi_{1}-\xi_{2}\right\|^{2}, \\
\left\|g^{I}\left(\xi_{1}\right)-g^{I}(\widetilde{z}(x, t))\right\|^{2} \leq h_{g}^{I}\left\|\xi_{1}-\xi_{2}\right\|^{2},
\end{array}\right.
$$

that hold for any $\xi_{1}, \xi_{2} \in \mathscr{H}_{n}^{1}(a, b)$.

Remark 2. Assumption 2 is more relaxed than the one in the literatures $[36,38]$ and does not need to know the bounds of the state. 
Assumption 3. The activation function term in equation (14) satisfies the following conditions:

$$
\left\{\begin{array}{l}
\operatorname{co}\left\{\underline{\underline{C}}, \bar{C}^{R}\right\} f^{R}(\widetilde{z}(x, t))-\operatorname{co}\left\{\underline{\underline{C}}, \bar{C}^{R}\right\} f^{R}(\widehat{z}(x, t)) \subseteq \operatorname{co}\left\{\underline{\underline{C}}, \bar{C}^{R}\right\}\left(f^{R}(\widetilde{z}(x, t))-f^{R}(\widehat{z}(x, t))\right), \\
\operatorname{co}\left\{\underline{\underline{C}}, \bar{C}^{I}\right\} f^{I}(\widetilde{z}(x, t))-\operatorname{co}\left\{\underline{\underline{C}}, \bar{C}^{I}\right\} f^{I}(\widehat{z}(x, t)) \subseteq \operatorname{co}\left\{\underline{C}, \bar{C}^{I}\right\}\left(f^{I}(\widetilde{z}(x, t))-f^{I}(\widehat{z}(x, t))\right), \\
\operatorname{co}\left\{\underline{\underline{C}}, \bar{C}^{R}\right\} f^{I}(\widetilde{z}(x, t))-\operatorname{co}\left\{\underline{R}, \bar{C}^{R}\right\} f^{I}(\widehat{z}(x, t)) \subseteq \operatorname{co}\left\{\underline{\underline{C}}, \bar{C}^{R}\right\}\left(f^{I}(\widetilde{z}(x, t))-f^{I}(\widehat{z}(x, t))\right), \\
\operatorname{co}\left\{\underline{\underline{C}}, \bar{C}^{I}\right\} f^{R}(\widetilde{z}(x, t))-\operatorname{co}\left\{\underline{I}, \bar{C}^{I}\right\} f^{R}(\widehat{z}(x, t)) \subseteq \operatorname{co}\left\{\underline{C}, \bar{C}^{I}\right\}\left(f^{R}(\widetilde{z}(x, t))-f^{R}(\widehat{z}(x, t))\right),
\end{array}\right.
$$

where $\widetilde{z}(x, t), \widehat{z}(x, t)$ are the solutions of equation (6) with the Dirichlet boundary condition.

Denote the error state, the error output, and the error activation function as $z^{R}(x, t)=\widehat{z}^{R}(x, t)-\widetilde{z}^{R}$ $(x, t), z^{I}(x, t)=\widehat{z}^{I}(x, t)-\widetilde{z}^{I} \quad(x, t), y^{R}(x, t)=\widehat{y}^{R}(x, t)-$ $\tilde{y}^{R}(x, t), y^{I}(x, t)=\hat{y}^{I}(x, t)-\tilde{y}^{I}(x, t), \quad H_{f}^{R}(\widehat{z}(x, t)-\quad \tilde{z}$ $(x, t))=f^{R}(\widehat{z}(x, t))-f^{R}(\widetilde{z}(x, t)), H_{f}^{I}(\widehat{z}(x, t)-\widetilde{z}(x, t))=$
$f^{I}(\widehat{z}(x, t))-f^{I}(\widetilde{z}(x, t))$, and $H_{\mathrm{g}}^{R}(\widehat{z}(x, t)-\widetilde{z}(x, t))=g^{R}$ $(\widehat{z}(x, t))-g^{R}(\widetilde{z}(x, t)), H_{g}^{I}(\widehat{z}(x, t)-\widetilde{z}(x, t))=g^{I}(\widehat{z}(x, t))-$ $g^{I}(\widetilde{z}(x, t))$.

Under Assumption 3, the error system of equation (14) can be rephrased as

$$
\left\{\begin{array}{l}
\frac{\partial z^{R}(x, t)}{\partial t} \in-\left(I_{N} \otimes A\right) z^{R}(x, t)+\left(I_{N} \otimes B\right) \frac{\partial^{2} z^{R}(x, t)}{\partial x^{2}}+c o\left\{\underline{\underline{C}}, \bar{C}^{R}\right\} H_{f}^{R}(z(x, t)) \\
-c o\left\{\underline{C}, \bar{C}^{I}\right\} H_{f}^{I}(z(x, t))+D \otimes \Gamma H_{g}^{R}(z(x, t-\tau(t)))+u^{R}(x, t), \\
y^{R}(x, t)=\left(I_{N} \otimes \beta\right) z^{R}(x, t), \\
\frac{\partial z^{I}(x, t)}{\partial t} \in-\left(I_{N} \otimes A\right) z^{I}(x, t)+\left(I_{N} \otimes B\right) \frac{\partial^{2} z^{I}(x, t)}{\partial x^{2}}+c o\left\{\underline{R}, \bar{C}^{R}\right\} H_{f}^{I}(z(x, t)) \\
+c o\left\{\underline{C}, \bar{C}^{I}\right\} H_{f}^{R}(z(x, t))+D \otimes \Gamma H_{g}^{R}(z(x, t-\tau(t)))+u^{I}(x, t), \\
y^{I}(x, t)=\left(I_{N} \otimes \beta\right) z^{I}(x, t) .
\end{array}\right.
$$


$\widetilde{C}^{R}(x, t) \in \operatorname{co}\left\{\underline{C}^{R}, \bar{C}^{R}\right\}, \widetilde{C}^{I}(x, t) \in \operatorname{co}\left\{\underline{C}^{I}, \bar{C}^{I}\right\}$, and one has

$$
\left\{\begin{array}{l}
\frac{\partial z^{R}(x, t)}{\partial t}=-\left(I_{N} \otimes A\right) z^{R}(x, t)+\left(I_{N} \otimes B\right) \frac{\partial^{2} z^{R}(x, t)}{\partial x^{2}}+\widetilde{C}^{R}(x, t) H_{f}^{R}(z(x, t)) \\
-\widetilde{C}^{I}(x, t) H_{f}^{I}(z(x, t))+D \otimes \Gamma H_{g}^{R}(z(x, t-\tau(t)))+u^{R}(x, t), \\
y^{R}(x, t)=\left(I_{N} \otimes \beta\right) z^{R}(x, t), \\
\frac{\partial z^{I}(x, t)}{\partial t}=-\left(I_{N} \otimes A\right) z^{I}(x, t)+\left(I_{N} \otimes B\right) \frac{\partial^{2} z^{I}(x, t)}{\partial x^{2}}+\widetilde{C}^{R}(x, t) H_{f}^{I}(z(x, t)) \\
+\widetilde{C}^{I}(x, t) H_{f}^{R}(z(x, t))+D \otimes \Gamma H_{g}^{R}(z(x, t-\tau(t)))+u^{I}(x, t), \\
y^{I}(x, t)=\left(I_{N} \otimes \beta\right) z^{I}(x, t),
\end{array}\right.
$$

with the initial values and the boundary values of networks as

$$
\left\{\begin{array}{l}
z(0, t)=z(l, t)=0, \quad t \in\left[t_{0}-\bar{\tau},+\infty\right] \\
z(x, t)=v(x, t) \\
v(x, t)=\widehat{v}(x, t)-\widetilde{v}(x, t) \\
(x, t) \in \Omega \times\left[t_{0}-\bar{\tau}, t_{0}\right]
\end{array}\right.
$$

Because the time-varying parameter is known, upper and lower bounds can be converted into a general semi-uncertain parameter by using the intermediate value theorem. There exists any $\varsigma_{i}(x, t)$ satisfied $0 \leq \varsigma_{i}(x, t) \leq 1$, and $\tilde{c}_{i}^{R}(x, t)=\underline{c}_{i}^{R}+\left(\bar{c}_{i}^{R}-\underline{c}_{i}^{R}\right) \varsigma_{i}^{R}(x, t), \tilde{c}_{i}^{I}(x, t)=\underline{c}_{i}^{I}+\left(\bar{c}_{i}^{I}-\underline{c}_{i}^{I}\right) \varsigma_{i}^{I}$ $(x, t)$. Then, $\widetilde{C}(x, t)$ varies over time and can be expressed as semi-uncertain parameters:

$$
\left\{\begin{array}{l}
\widetilde{C}^{R}(x, t)=\underline{R}+\left(\bar{C}^{R}-\underline{R}\right) F^{R}(x, t), \\
\widetilde{C}^{I}(x, t)=\underline{I}+\left(\bar{C}^{I}-\underline{I}\right) F^{I}(x, t)
\end{array}\right.
$$

where $\quad F^{T}(x, t) F(x, t) \leq I_{n N}, \quad F^{R}(x, t)=\operatorname{diag}\left(F_{1}^{R}\right.$ $\left.(x, t), \ldots, F_{N}^{R}(x, t)\right), F_{i}^{R}(x, t)=\operatorname{diag}\left(\varsigma_{1}^{R}(x, t), \ldots, \varsigma_{n}^{R}(x, t)\right)$, $F^{I}(x, t)=\operatorname{diag}\left(F_{1}^{I}(x, t), \ldots, F_{N}^{I}(x, t)\right), F_{i}^{I}(x, t)=\operatorname{diag}$ $\left(\varsigma_{1}^{I}(x, t), \ldots, \varsigma_{n}^{I}(x, t)\right)$, and $O^{R}=\left(\bar{C}^{R}-\underline{C}^{R}\right), O^{I}=\left(\bar{C}^{I}-\underline{C}^{I}\right)$.

Remark 3. Equations (18) and (20) can be seen as realvalued error models with semi-uncertainty parameters.

\section{Output Event-Triggered Synchronization of CMRDNNs}

3.1. Piecewise Event-Triggered Control Strategy. The state feedback input controller of the slave networks system can be designed as follows.

$$
u_{i}(x, t)=-\sum_{q=1}^{Q} u_{i}\left(x_{q}, t\right)=-\sum_{q=1}^{Q} \alpha\left(x_{q}\right) L_{i} \beta^{\mathrm{T}} \beta z_{i}\left(x_{q}, t_{k}^{i}\right),
$$

where $L_{i}=\operatorname{diag}\left\{L_{i 1}, L_{i 2}, \ldots, L_{i n}\right\}, \mathrm{i} \in \mathcal{N}_{n} ; \alpha\left(x_{q}\right)$ denotes a spatial distributed operator with $\int_{\Omega} z(x, t) \alpha\left(x_{q}\right)$ $\mathrm{d} x=z\left(x_{q}, t\right)$, and $\mathrm{Q}$ represents the number of the spatial sampled-data. For simplicity, $\alpha\left(x_{q}\right)$ can be replaced by $\alpha$.

Remark 4. $u_{i}\left(x_{q}, t\right)$ only uses the output state in the partial spatial domain $x_{q}$ and at some discrete time instantst $t_{k}$. Thus, $u_{i}\left(x_{q}, t\right)$ can be seen as a distributed spatiotemporal sampled-data controller. Different from the spatial sampled-data controller designed in [17], this controller designed in equation (21) is a spatial and temporal sampling control.

$$
\text { Let } \quad u(x, t)=\left[u_{1}^{T}(x, t), u_{2}^{T}(x, t), \ldots, u_{N}^{T}(x, t)\right]^{T} \text {, }
$$
$L=\operatorname{diag}\left\{L_{1}, L_{2}, \ldots, L_{N}\right\}$, and $u(x, t)$ can be formulated into the real-valued compact matrix form:

$$
\left\{\begin{array}{l}
u^{R}(x, t)=-\sum_{q=1}^{Q} \alpha L^{R}\left(I_{N} \otimes \beta^{T} \beta\right) z^{R}\left(x_{q}, t_{k}\right), \\
u^{I}(x, t)=-\sum_{q=1}^{Q} \alpha L^{I}\left(I_{N} \otimes \beta^{T} \beta\right) z^{I}\left(x_{q}, t_{k}\right),
\end{array}\right.
$$


where $z^{R}\left(x_{q}, t_{k}\right)=\left(z_{1}^{R T}\left(x_{q}, t_{k}^{1}\right), \ldots, z_{N_{T}}^{R T}\left(x_{q}, t_{k}^{N}\right)\right)^{T}$, and $z^{I}\left(x_{q}, t_{k}\right)=\left(z_{1}^{I T}\left(x_{q}, t_{k}^{1}\right), \ldots, z_{N}^{I T}\left(x_{q}, t_{k}^{N}\right)\right)^{T}$.

The transmission time sequence of the input controller can be determined by event-triggered conditions.

The information flow diagram of the distributed eventtriggered control mechanism corresponding to the $i^{\text {th }}$ slave system is shown in Figure 1, where the event trigger is used to determine whether the controller updates with the current spatial sampling signal. That is, an update event is determined by the event-triggered condition [44]:

The distributed output event-triggered condition is designed as

$$
t_{k+1}^{i}=\inf \left\{t: t \geq t_{k}^{i}, \sum_{q=1}^{Q}\left(\beta e_{i}\left(x_{q}, t\right)\right)^{T} \beta e_{i}\left(x_{q}, t\right)-\sum_{q=1}^{Q} \gamma_{i}\left(\beta z_{i}\left(x_{q}, t\right)\right)^{T} \beta z_{i}\left(x_{q}, t\right)-\bar{\eta}_{i}(t)>0\right\},
$$

where $e_{i}\left(x_{q}, t\right)=z_{i}\left(x_{q}, t_{k}^{i}\right)-z_{i}\left(x_{q}, t\right) ; t_{k+1}^{i}$ denotes the next event-triggered time instants of the $i^{\text {th }}$ networks; and $\gamma_{i}, \bar{\eta}_{i}(t)>0$ represent parts of the event trigger which can be adaptive derived later.
The condition of equation (23) can generate a triggering time sequence $\left\{t_{0}^{i}, \cdots, t_{k}^{i}, t_{k+1}^{i}, \cdots\right\}$ for the $i^{\text {th }}$ networks control.

Let $\quad e(x, t)=\left(e_{1}^{T}(x, t), \ldots, e_{N}^{T}(x, t)\right)^{T}$, $\Upsilon=\operatorname{diag}\left\{\gamma_{1}, \ldots \gamma_{\mathrm{N}}\right\}$, and the real-valued compact form of event-triggered condition can be expressed as

$$
\left\{\begin{array}{l}
\sum_{q=1}^{Q} e^{R T}\left(x_{q}, t\right)\left(I_{N} \otimes \beta^{T} \beta\right) e^{R}\left(x_{q}, t\right)>\sum_{q=1}^{Q} z^{R T}\left(x_{q}, t\right)\left(\Upsilon \otimes \beta^{T} \beta\right) z^{R}\left(x_{q}, t\right)+\bar{\eta}^{R}(t), \\
\sum_{q=1}^{Q} e^{I T}\left(x_{q}, t\right)\left(I_{N} \otimes \beta\right) e^{I}\left(x_{q}, t\right)>\sum_{q=1}^{Q} z^{I T}\left(x_{q}, t\right)(\Upsilon \otimes \beta) z^{I}\left(x_{q}, t\right)+\bar{\eta}^{I}(t),
\end{array}\right.
$$

where $\quad \bar{\eta}(t)=\bar{\eta}^{R}(t)+\bar{\eta}^{I}(t), \quad \bar{\eta}^{R}(t)=\sum_{i=1}^{N} \bar{\eta}_{i}^{R}(t)$, and $\bar{\eta}^{I}$ $(t)=\sum_{i=1}^{N} \bar{\eta}_{i}^{I}(t)$.

$$
\bar{\eta}_{i}(t)=\left(\bar{\eta}_{i 0}^{R}+\bar{\eta}_{i 0}^{I}\right) f f(t), f f(t)=\left\{\begin{array}{l}
\exp \left(-\kappa_{1}\left(t-t_{0}\right)\right), t_{0}<t<t_{f} \\
\exp \left(-\kappa_{1}\left(t_{f}-t_{0}\right)\right), \quad t \geq t_{f}
\end{array}\right.
$$

where four parameters $\bar{\eta}_{i 0}^{R}, \bar{\eta}_{i 0}^{I}, \bar{\eta}_{i 0}^{R}$, and $\bar{\eta}_{i 0}^{I}$ are the positives, $\bar{\eta}_{0}^{R}=\sum_{i=1}^{N} \bar{\eta}_{i}^{R}\left(t_{0}\right)$, and $\bar{\eta}_{0}^{I}=\sum_{i=1}^{N} \bar{\eta}_{i}^{I}\left(t_{0}\right)$.

From equation (25), we find that $t_{k+1}^{i}>t_{k}^{i}$ is always satisfied. Therefore, the Zeno behaviour can be naturally excluded.

Remark 5. The distributed event-triggered method in [38] defined an event trigger for each neural network, and each event trigger required the entire state information of the corresponding neural networks. Different from the definition in [38], the distributed event trigger defined in equation (14) uses spatial sampled-data, which reduces the communication load and decreases the measurement cost.

Remark 6. Because the semi-uncertain error system always has a certain range of error [25], quasisynchronization is a more realistic goal. Meanwhile, to better solving the Zeno problem, the function $\bar{\eta}_{i}(t)$ in the event-triggered condition must be positive. Therefore, the piecewise function $\bar{\eta}_{i}(t)$ in equation (25) is a good selection which is different from the exponential function in literatures $[45,46]$.

Using the above distributed output piecewise eventtriggered control strategy (equations (24) and (25)), the quasisynchronization conditions are obtained, and a theorem is constructed for the master-slave networks system in Section 3.2.

\subsection{Synchronization Analysis}

Theorem 1. Under Assumptions 1, 2, and 3, using the distributed piecewise event-triggered mechanism (equation (23)) and the sampling controller (equation (21)), the master-slave networks systems (equations (1) and (3)) can be output quasisynchronized within the error $\Theta$, and the error system (equation (18)) converges exponentially to the error level $\Theta$ with the convergence index $\underline{\kappa}_{2}>0$, if the criteria are satisfied: 


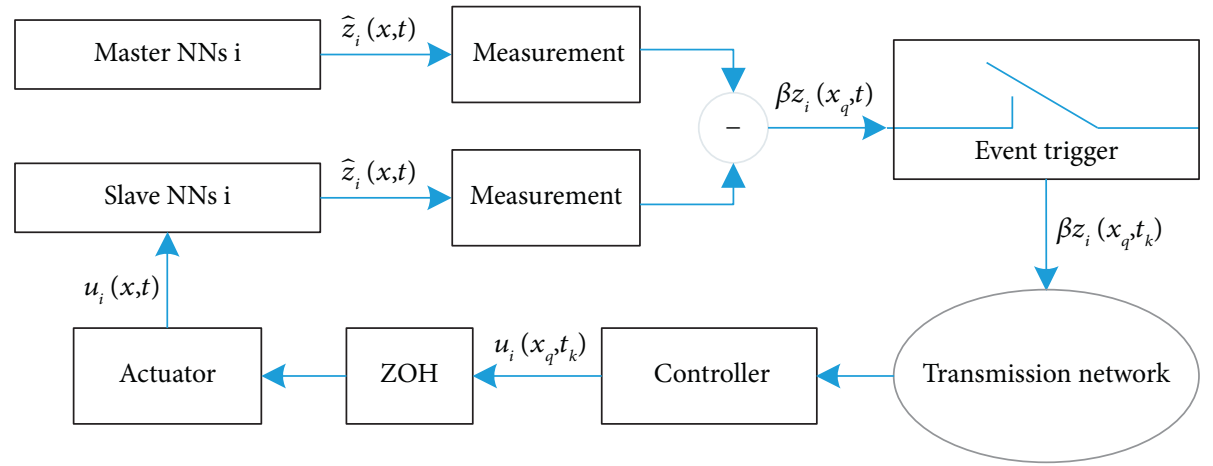

FIgURE 1: The $i^{\text {th }}$ event-triggered controller structure of the $i^{\text {th }}$ master-slave system.

$$
\left\{\begin{array}{l}
\frac{1}{\varepsilon^{2}}(2 \varepsilon-1)>\Upsilon \\
\lambda_{1}>\lambda_{2} \exp \left(\kappa_{2}(t) \tau(t)\right)>0
\end{array}\right.
$$

where

$$
\begin{aligned}
& \Theta=\frac{\varepsilon}{\lambda_{1}-\lambda_{2}}\left(\lambda_{\max }\left(L^{R}\right) \bar{\eta}_{0}^{R}+\lambda_{\max }\left(L^{I}\right) \bar{\eta}_{0}^{I}\right) \sup _{t \geq t_{0}} f f(t), \\
& f f(t)= \begin{cases}\exp \left(-\kappa_{1}\left(t-t_{0}\right)\right), & t_{0}<t<t_{f}, \\
\exp \left(-\kappa_{1}\left(t_{f}-t_{0}\right)\right), & t \geq t_{f},\end{cases} \\
& \underline{\kappa}_{2}=\inf _{t_{0} \leq t}\left\{\kappa_{2}(t): \kappa_{2}(t)-\lambda_{1}+\lambda_{2} \exp \left(\kappa_{2}(t) \tau(t)\right)=0\right\} \text {, } \\
& \lambda_{1}=\lambda_{\min }\left\{\left(2\left(I_{N} \otimes A\right)+\mathscr{R}_{1 R}-\frac{3}{\varepsilon} I_{N n}-\varepsilon h_{f}^{R}\left(\mathscr{P}_{1 R}+\mathscr{P}_{1 I}\right)\right), 2\left(I_{N} \otimes A\right)+\mathscr{R}_{1 I}-\frac{3}{\varepsilon} I_{N n}-\varepsilon h_{f}^{I}\left(\mathscr{P}_{1 R}+\mathscr{P}_{1 I}\right)\right\}, \\
& \lambda_{2}=\lambda_{\max }\left\{\left(I_{N} \otimes \beta^{T} \beta\right) \varepsilon h_{g}^{R} \mathscr{P}_{2},\left(I_{N} \otimes \beta^{T} \beta\right) \varepsilon h_{g}^{I} \mathscr{P}_{2}\right\} \\
& \mathscr{R}_{1 R}=M_{1} M_{2} L^{R}\left(M_{1}+2 \xi_{1} M_{2} L^{R}\right)^{-1} \\
& \mathscr{R}_{1 I}=M_{1} M_{2} L^{I}\left(M_{1}+2 \xi_{1} M_{2} L^{I}\right)^{-1} \text {, } \\
& \mathscr{P}_{1 R}=C^{R T} C^{R}+O^{R T} O^{R}, \\
& \mathscr{P}_{1 I}=C^{I T} C^{I}+O^{I T} O^{I}, \\
& \mathscr{P}_{2}=\left(D^{T} D \otimes \Gamma^{T} \Gamma\right) \\
& M_{1}=2\left(I_{N} \otimes B\right), \\
& M_{2}=\left(\left(2-\frac{1}{\varepsilon}\right) I_{N}-\varepsilon \Upsilon\right) \otimes I_{n}, \\
& \xi_{1}=\frac{1}{\pi^{2}}\left(\max _{2 \leq q \leq Q}\left\{x_{q}-x_{q-1}, x_{1},\left(l-x_{Q}\right)\right\}\right)^{2} \text {, }
\end{aligned}
$$

where $\lambda_{\min }$ and $\lambda_{\max }$ mean the minimum and maximum eigenvalues, respectively.

Proof. Construct the following Lyapunov functional for the system (equation (18)) as

$$
\begin{aligned}
V(t)= & \int_{\Omega} z^{R T}(x, t)\left(I_{N} \otimes \beta^{T}\right)\left(I_{N} \otimes \beta\right) z^{R}(x, t) \mathrm{d} x \\
& +\int_{\Omega} z^{I T}(x, t)\left(I_{N} \otimes \beta^{T}\right)\left(I_{N} \otimes \beta\right) z^{I}(x, t) \mathrm{d} x .
\end{aligned}
$$


By using integral formula of division and equation (18), we can derive

$$
\begin{gathered}
\int_{\Omega} z^{R T}(x, t) 2\left(I_{N} \otimes \beta^{T} \beta B\right) \frac{\partial^{2} z^{R}(x, t)}{\partial x^{2}} \mathrm{~d} x \leq-\int_{\Omega} \frac{\partial z^{R T}(x, t)}{\partial x} 2\left(I_{N} \otimes \beta^{T} \beta B\right) \frac{\partial z^{R}(x, t)}{\partial x} \mathrm{~d} x, \\
\int_{\Omega} z^{I T}(x, t) 2\left(I_{N} \otimes \beta^{T} \beta B\right) \frac{\partial^{2} z^{I}(x, t)}{\partial x^{2}} \mathrm{~d} x \leq-\int_{\Omega} \frac{\partial z^{I T}(x, t)}{\partial x} 2\left(I_{N} \otimes \beta^{T} \beta B\right) \frac{\partial z^{I}(x, t)}{\partial x} \mathrm{~d} x .
\end{gathered}
$$

According to equation (24) and by using Cauchy inequality yields

$$
\begin{aligned}
& -2 \int_{\Omega} z^{R T}(x, t)\left(I_{N} \otimes \beta^{T} \beta\right) \sum_{q=1}^{Q} \alpha\left(I_{N} \otimes \beta^{T} \beta\right) L^{R} z^{R}\left(x_{q}, t_{k}\right) \mathrm{d} x \\
& =-2 \sum_{q=1}^{Q} z^{R T}\left(x_{q}, t\right)\left(I_{N} \otimes \beta^{T} \beta\right) L^{R}\left(e^{R}\left(x_{q}, t\right)+z^{R}\left(x_{q}, t\right)\right) \\
& \leq \sum_{q=1}^{Q}\left(\left(\frac{1}{\varepsilon}-2\right) z^{R T}\left(x_{q}, t\right)\left(I_{N} \otimes \beta^{T} \beta\right) L^{R} z^{R}\left(x_{q}, t\right)+\varepsilon e^{R T}\left(x_{q}, t\right)\left(I_{N} \otimes \beta^{T} \beta\right) L^{R} e^{R}\left(x_{q}, t\right)\right) \\
& =-\sum_{q=1}^{Q} z^{R T}\left(x_{q}, t\right)\left(\left(2-\frac{1}{\varepsilon}\right) I_{N}-\varepsilon \Upsilon\right) \otimes\left(\beta^{T} \beta\right) L^{R} z^{R}\left(x_{q}, t\right)+\eta^{R}(t) .
\end{aligned}
$$

Similarly,

$$
\begin{gathered}
-2 \sum_{q=1}^{Q} z^{I T}\left(x_{q}, t\right)\left(I_{N} \otimes \beta^{T} \beta\right) L^{I} z^{I}\left(x_{q}, t_{k}\right) \\
\leq-\sum_{q=1}^{Q} z^{I T}\left(x_{q}, t\right)\left(\left(2-\frac{1}{\varepsilon}\right) I_{N}-\varepsilon \Upsilon\right) \\
\otimes\left(\beta^{T} \beta\right) L^{I} z^{I}\left(x_{q}, t\right)+\eta^{I}(t) .
\end{gathered}
$$

where $\eta^{R}(t)=\varepsilon \lambda_{m}\left(L^{R}\right) \bar{\eta}^{R}(t)$ and $\eta^{I}(t)=\varepsilon \lambda_{m}\left(L^{I}\right) \bar{\eta}^{I}(t)$.

$$
\begin{aligned}
-\int_{\Omega} & \frac{\partial z^{R T}(x, t)}{\partial x} 2\left(I_{N} \otimes \beta^{T} \beta B\right) \frac{\partial z^{R}(x, t)}{\partial x} \mathrm{~d} x \\
& \quad-\sum_{q=1}^{Q} z^{R T}\left(x_{q}, t\right)\left(\left(2-\frac{1}{\varepsilon}\right) I_{N}-\varepsilon \Upsilon\right) \otimes\left(\beta^{T} \beta\right) L^{R} z^{R}\left(x_{q}, t\right) \\
\leq & -\int_{\Omega} z^{R T}(x, t)\left(I_{N} \otimes \beta^{T} \beta\right) \mathscr{R}_{1 R} z^{R}(x, t) \mathrm{d} x .
\end{aligned}
$$

Similarly,

According to Lemma 1, Corollary 1 , and the boundary condition of equation (18),

$$
\begin{aligned}
& -\int_{\Omega} \frac{\partial z^{I T}(x, t)}{\partial x} 2\left(I_{N} \otimes \beta^{T} \beta B\right) \frac{\partial z^{I}(x, t)}{\partial x} \mathrm{~d} x-\sum_{q=1}^{Q} z^{I T}\left(x_{q}, t\right)\left(\left(2-\frac{1}{\varepsilon}\right) I_{N}-\varepsilon \Upsilon\right) \otimes\left(\beta^{T} \beta\right) L^{I} z^{I}\left(x_{q}, t\right) \\
& \quad \leq-\int_{\Omega} z^{I T}(x, t)\left(I_{N} \otimes \beta^{T} \beta\right) \mathscr{R}_{1 I} z^{I}(x, t) \mathrm{d} x,
\end{aligned}
$$

where $\mathscr{R}_{1 R}=M_{1} M_{2} L^{R}\left(M_{1}+2 \xi_{1} M_{2} L^{R}\right)^{-1}, \quad \mathscr{R}_{1 I}=M_{1} M_{2}$ $L^{I}\left(M_{1}+2 \xi_{1} M_{2} L^{I}\right)^{-1}, \quad M_{1}=2\left(I_{N} \otimes B\right), \quad \Upsilon<\left(1 / \varepsilon^{2}\right)(2 \varepsilon-1)$
$I_{N}, \quad M_{2}=\left((2-(1 / \varepsilon)) I_{N}-\varepsilon \Upsilon\right) \otimes I_{n}$, $\operatorname{and} \xi_{1}=\left(1 / \pi^{2}\right)$ 
According to Assumption 2 and Cauchy inequality, the real part and imaginary part of the activation function can be derived:

$$
\left\{\begin{array}{l}
2 \int_{\Omega} z^{R T}(x, t)\left(I_{N} \otimes \beta^{T} \beta\right) \widetilde{C}^{R}(x, t) \mathrm{H}_{f}^{R}(z(x, t)) \mathrm{d} x-2 \int_{\Omega} z^{R T}(x, t)\left(I_{N} \otimes \beta^{T} \beta\right) \widetilde{C}^{I}(x, t) \mathrm{H}_{f}^{I}(z(x, t)) \mathrm{d} x \\
\leq \int_{\Omega} z^{R T}(x, t)\left(I_{N} \otimes \beta^{T} \beta\right)\left(\frac{2}{\varepsilon} I_{N n}+\varepsilon h_{f}^{R} \mathscr{P}_{1 R}\right) z^{R}(x, t) \mathrm{d} x+\int_{\Omega} z^{I T}(x, t)\left(I_{N} \otimes \beta^{T} \beta\right)\left(\varepsilon h_{f}^{I} \mathscr{P}_{1 I}\right) z^{I}(x, t) \mathrm{d} x \\
2 \int_{\Omega} z^{I T}(x, t)\left(I_{N} \otimes \beta^{T} \beta\right)\left(\widetilde{C}^{R}(x, t) \mathrm{H}_{f}^{I}(z(x, t))+\widetilde{C}^{I}(x, t) \mathrm{H}_{f}^{R}(z(x, t))\right) \mathrm{d} x \\
\leq \int_{\Omega} z^{I T}(x, t)\left(I_{N} \otimes \beta^{T} \beta\right)\left(\frac{2}{\varepsilon} I_{N n}+\varepsilon h_{f}^{I} \mathscr{P}_{1 R}\right) z^{I}(x, t) \mathrm{d} x+\int_{\Omega} z^{R T}(x, t)\left(I_{N} \otimes \beta^{T} \beta\right)\left(\varepsilon h_{f}^{R} \mathscr{P}_{1 I}\right) z^{R}(x, t) \mathrm{d} x
\end{array}\right.
$$

where $\mathscr{P}_{1 R}=C^{R T} C^{R}+O^{R T} O^{R}$ and $\mathscr{P}_{1 I}=C^{I T} C^{I}+O^{I T} O^{I}$.

Real part and imaginary part of the state-coupling part can be processed as follows:

$$
\left\{\begin{array}{l}
2 \int_{\Omega} z^{R T}(x, t)\left(I_{N} \otimes \beta^{T} \beta\right)(D \otimes \Gamma) \mathrm{H}_{g}^{R}(z(x, t-\tau(t))) \mathrm{d} x \\
\leq \int_{\Omega} z^{R T}(x, t) \frac{1}{\varepsilon}\left(I_{N} \otimes \beta^{T} \beta\right) z^{R}(x, t) \mathrm{d} x+\int_{\Omega} z^{R T}(x, t-\tau(t))\left(I_{N} \otimes \beta^{T} \beta\right) \varepsilon h_{g}^{R} \mathscr{P}_{2} z^{R}(x, t-\tau(t)) \mathrm{d} x \\
2 \int_{\Omega} z^{I T}(x, t)\left(I_{N} \otimes \beta^{T} \beta\right)(D \otimes \Gamma) \mathrm{H}_{g}^{I}(z(x, t-\tau(t))) \mathrm{d} x \\
\leq \int_{\Omega} z^{I T}(x, t) \frac{1}{\varepsilon}\left(I_{N} \otimes \beta^{T} \beta\right) z^{I}(x, t) \mathrm{d} x+\int_{\Omega} z^{I T}(x, t-\tau(t))\left(I_{N} \otimes \beta^{T} \beta\right) \varepsilon h_{g}^{I} \mathscr{P}_{2} z^{I}(x, t-\tau(t)) \mathrm{d} x
\end{array}\right.
$$

where $\mathscr{P}_{2}=\left(D^{T} D \otimes \Gamma^{T} \Gamma\right)$.

According to equation (28) to equation (35),

$$
\begin{aligned}
\dot{V}(t)= & 2 \int_{\Omega} z^{R T}(x, t)\left(\mathrm{I}_{N} \otimes \beta^{\mathrm{T}} \beta\right) \frac{\partial z^{R}(x, t)}{\partial t} \mathrm{~d} x+2 \int_{\Omega} z^{I T}(x, t)\left(I_{N} \otimes \beta^{\mathrm{T}} \beta\right) \frac{\partial z^{I}(x, t)}{\partial t} \mathrm{~d} x \\
\leq & \int_{\Omega} z^{R T}(x, t)\left(I_{N} \otimes \beta^{\mathrm{T}} \beta\right)\left(-2\left(\mathrm{I}_{N} \otimes A\right)-\mathscr{R}_{1 R}+\frac{3}{\mathcal{E}} I_{N n}+\varepsilon \mathrm{h}_{f}^{R}\left(\mathscr{P}_{1 R}+\mathscr{P}_{1 I}\right)\right) z^{R}(x, t) \\
& +\int_{\Omega} z^{I T}(x, t)\left(I_{N} \otimes \beta^{\mathrm{T}} \beta\right)\left(-2\left(I_{N} \otimes A\right)-\mathscr{R}_{1 I}+\frac{3}{\varepsilon} I_{N n}+\varepsilon \mathrm{h}_{f}^{I}\left(\mathscr{P}_{1 R}+\mathscr{P}_{1 I}\right)\right) z^{I}(x, t) \mathrm{d} x \\
& +\int_{\Omega} z^{R T}(x, t-\tau(t))\left(I_{N} \otimes \beta^{\mathrm{T}} \beta\right) \varepsilon h_{g}^{R} \mathscr{P}_{2} z^{R}(x, t-\tau(t)) \mathrm{d} x \\
& +\int_{\Omega} z^{I T}(x, t-\tau(t))\left(I_{N} \otimes \beta^{\mathrm{T}} \beta\right) \varepsilon h_{g^{I}} \mathscr{P}_{2} z^{I}(x, t-\tau(t)) \mathrm{d} x \\
& +\varepsilon\left(\lambda_{m}\left(L^{R}\right) \bar{\eta}^{R}(t)+\lambda_{m}\left(L^{I}\right) \bar{\eta}^{I}(t)\right) \\
\leq & -\lambda_{1} V(t)+\lambda_{2} V(t-\tau(t))+\varepsilon\left(\lambda_{\max }\left(L^{R}\right) \bar{\eta}^{R}(t)+\lambda_{\max }\left(L^{I}\right) \bar{\eta}^{I}(t)\right),
\end{aligned}
$$


with $\lambda_{0}, \lambda_{1}, \lambda_{2}$ defined in Theorem 1 .

From Lemma 2, we can obtain

$$
V(t) \leq \Theta+\sup _{t-\tau(t) \leq s \leq t} V(s) \exp \left(-\underline{\kappa}_{2}\left(t-t_{0}\right)\right),
$$

where $\Theta=\left(\varepsilon /\left(\lambda_{1}-\lambda_{2}\right)\right)\left(\lambda_{\max }\left(L^{R}\right) \bar{\eta}_{0}^{R}+\lambda_{\max }\left(L^{I}\right) \bar{\eta}_{0}^{I}\right) \sup _{t \geq t_{0}}$ $f f(t), \underline{\kappa}_{2}=\inf _{t_{0} \leq t}\left\{\kappa_{2}(t): \quad \kappa_{2}(t)-\lambda_{1}+\lambda_{2} \exp \quad\left(\kappa_{2}(t) \tau\right.\right.$ $(t))=0\}$, and $\lambda_{1}>\lambda_{2} \exp \left(\kappa_{2}(t) \tau(t)\right)$.

If $f f(t)$ is set as equation (25) and $\kappa_{1} \geq \underline{\kappa}_{2}$, then the error system in equations (18) and (20) converges exponentially to $\Theta$ with the exponential index $\underline{\kappa}_{2}$. That is, the master-slave system (equations (1) and (3)) achieves quasisynchronization within the error level $\Theta$.

\section{Numerical Examples}

This section mainly completes multiple numerical simulations to testify the theoretical results.

Consider the master-slave error networks system as

$$
\begin{aligned}
\frac{\partial z_{i}(x, t)}{\partial t}= & -A z_{i}(x, t)+B \frac{\partial^{2} z_{i}(x, t)}{\partial x^{2}} \\
& +c_{i}\left(z_{i}(x, t)\right) f_{i}\left(z_{i}(x, t)\right)+\sum_{j=1}^{N} d_{i j} \Gamma g_{j} \\
& \cdot\left(z_{j}\left(x, t-\tau_{j}(t)\right)\right)-\sum_{q=1}^{Q} \alpha\left(x_{q}\right) L_{i} \beta^{T} \beta z_{i}\left(x_{q}, t_{k}\right),
\end{aligned}
$$

where $i=1,2, N=2, n=3, g_{i}(\cdot)=f_{i}(\cdot)=\tan h(\cdot)$;

$$
\begin{aligned}
& c_{1}(v(\cdot, s))= \begin{cases}-0.5-1.2 l, & v(\cdot, s) \downarrow, \\
-0.4-1.1 \iota & v(\cdot, s) \uparrow, \\
\lim _{s \longrightarrow t^{-}}(v(\cdot, s)) & v(\cdot, s) \text { unchange, }\end{cases} \\
& c_{2}(v(\cdot, s))= \begin{cases}-1-1 \iota, & v(\cdot, s) \downarrow, \\
-0.9-0.9 l, & v(\cdot, s) \uparrow, \\
\lim _{s \longrightarrow t^{-}}(v(\cdot, s)), & v(\cdot, s) \text { unchange, }\end{cases}
\end{aligned}
$$

$$
\begin{aligned}
A & =\left[\begin{array}{ccc}
0.1 & 0 & 0 \\
0 & 0.1 & 0 \\
0 & 0 & 0.1
\end{array}\right], \\
B & =\left[\begin{array}{ccc}
0.5 & 0 & 0 \\
0 & 0.5 & 0 \\
0 & 0 & 0.5
\end{array}\right], \\
D & =\left[\begin{array}{cc}
-0.3 & 0.3 \\
0.3 & -0.3
\end{array}\right], \\
\Gamma & =\left[\begin{array}{ccc}
0.5 & 0 & 0 \\
0 & 0.5 & 0 \\
0 & 0 & 0.5
\end{array}\right] .
\end{aligned}
$$

The positions of two spatial point measurements are $x_{1}=$ $0.25 l, x_{2}=0.75 l$. The initial conditions of the master system are

$$
\begin{aligned}
& \widetilde{z}_{1}(x, 0)=\left(\begin{array}{c}
(2-2 \cos (3 \pi x))+i(1-\cos (4 \pi x)) \\
(1-3 \sin (5 \pi x))+i(2-3 \sin (4 \pi x)) \\
(-1.5+0.5 \sin (4 \pi x))+i(-0.5+\sin (3 \pi x))
\end{array}\right), \\
& \widetilde{z}_{2}(x, 0)=\left(\begin{array}{c}
\cos (5 \pi x)+i(1+2 \cos (6 \pi x)) \\
(3-3 \sin (3 \pi x))+i(4-\sin (2 \pi x)) \\
(1.5+2 \sin (2 \pi x))+i(3+3 \sin (2 \pi x))
\end{array}\right) .
\end{aligned}
$$

The initial conditions of the slave system are

$$
\left\{\begin{array}{l}
\widehat{z}_{1}(x, 0)=((3 \cos (2 \pi x)-2)+(4 \cos (\pi x)-3) \iota, 3+1.5 \iota, 1+1 \iota)^{T}, \\
\widehat{z}_{2}(x, 0)=(5 \cos (7 \pi x)-3+(5 \cos (7 \pi x)-3) \iota, 1+1 \iota, 1+1 \iota)^{T} .
\end{array}\right.
$$

4.1. Output Static Event-Triggered Control (OSETC) with $\beta=(100 ; 010)$. Event trigger's parameters satisfied with equation (26) are set as $\gamma_{i}=0.02,, \bar{\eta}_{i}(t)=0.01$, and $L_{i j}=10$. And the simulation results are shown in Figures 2-4. Figure 2 represents the $j^{\text {th }}$ dimensional state norm evolution of the $i^{\text {th }}$ error networks over time. The real-valued state norm evolution and the imaginary-valued one can be formulated as

$$
\left\{\begin{array}{l}
\left\|z_{i j}^{R}(x, t)\right\|^{2}=\int_{\Omega} z_{i j}^{R T}(x, t), z_{i j}^{R}(x, t) \mathrm{d} x, \\
\left\|z_{i j}^{I}(x, t)\right\|^{2}=\int_{\Omega} z_{i j}^{I T}(x, t), z_{i j}^{I}(x, t) \mathrm{d} x, \quad i=1,2, j=1,2,3 .
\end{array}\right.
$$

Figures 2-4 show that the first dimension and the second dimension of the error state converge over time, while the third dimension does not converge. That is, the output synchronization has been realized which matches the output matrix. Figure 5 shows that the four triggered time series are different which are consistent with their distribution characteristics of the four event triggers.

4.2. Output Piecewise Event-Triggered Control (OPETC) with $\beta=(100 ; 010 ; 001)$. The output matrix $\beta$ is a unit matrix, and the output states are common full-dimensional states. Event trigger's parameters are set as $\gamma_{i}=0.02$, $\bar{\eta}_{i}(t)=0.01 \exp (-0.5 t)$ for $(t \leq 7), \bar{\eta}_{i}(t)=0.01 \exp (-3.5)$ for $(t>7)$, and the input control gain is $L_{i j}=10$. These parameters are satisfied with equation (26), and the simulation results are shown in Figures 6 and 7. Figure 6 indicates that the real part (Figure 6(a)) and the imaginary part (Figure 6(b)) of the error state converge over time. While, Figure 7 denotes that the four triggered time series that are independent of each other.

4.3. Multistrategy Comparison. The simulations of output static event-triggered control (OSETC), output dynamic event-triggered control (ODETC), and output time- 


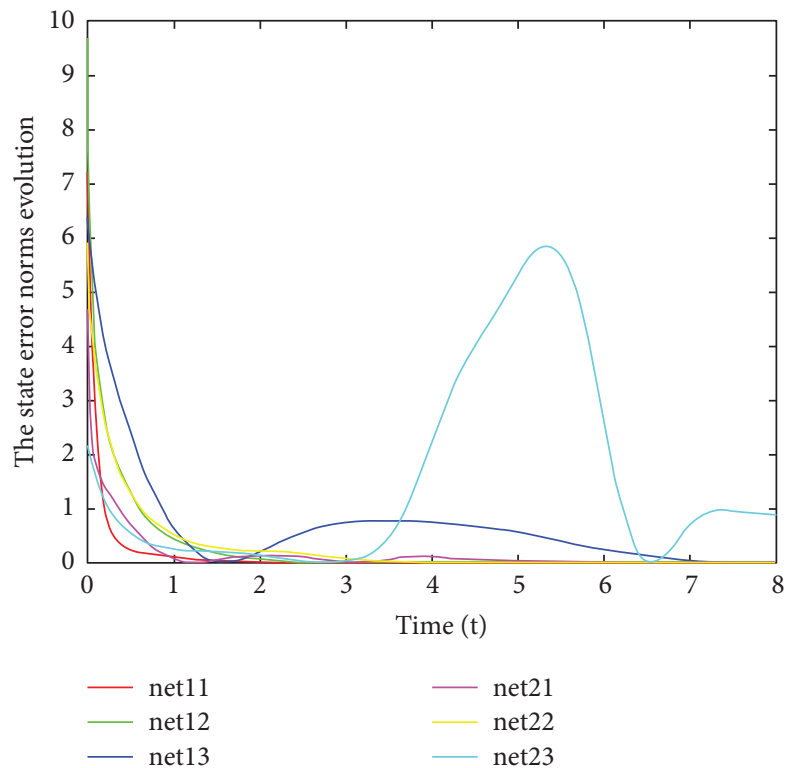

(a)

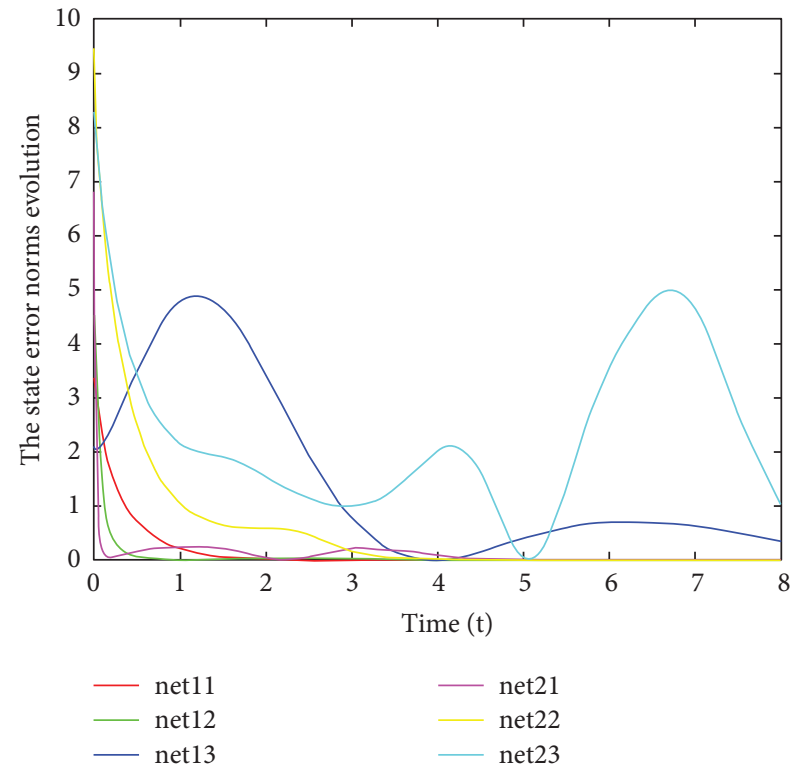

(b)

Figure 2: Real part and imaginary part of state error norm evolution under the output static event-triggered control. net11, net12, and net13 represent three-dimensional state norm evolution of the 1st error network. net21, net22, and net23 represent three-dimensional state norm evolution of the 2nd error network. (a) Real part of state error evolution. (b) Imaginary part of state error evolution.

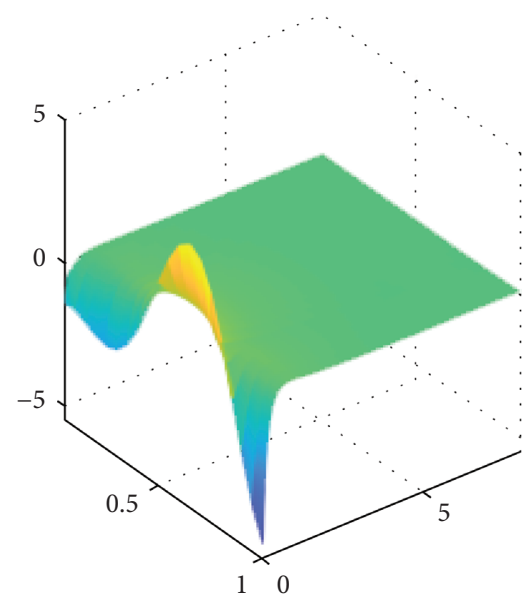

(a)

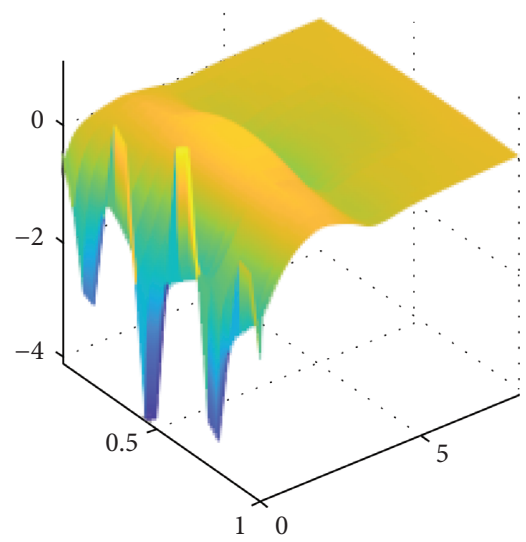

(d)

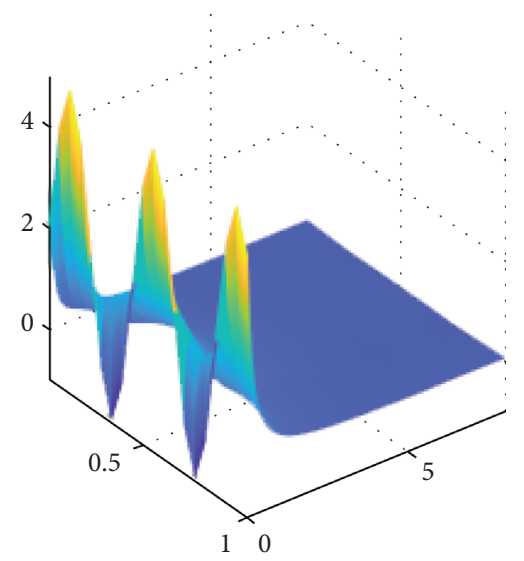

(b)

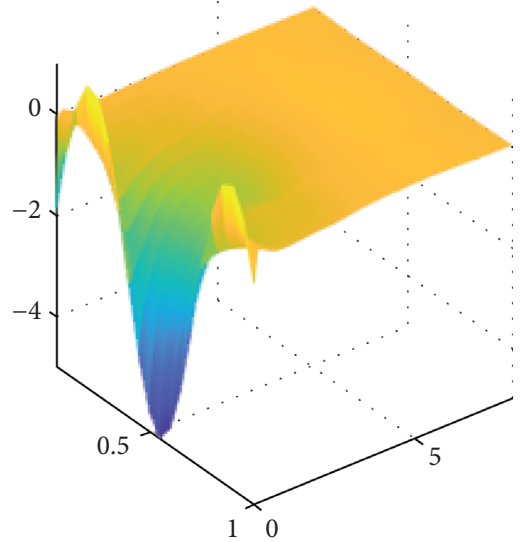

(e)

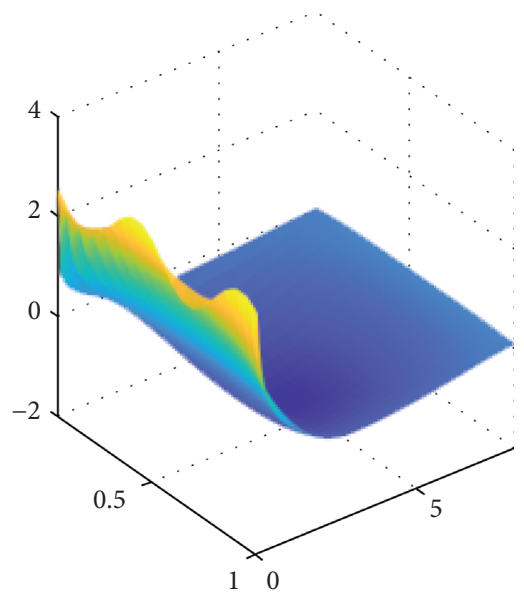

(c)

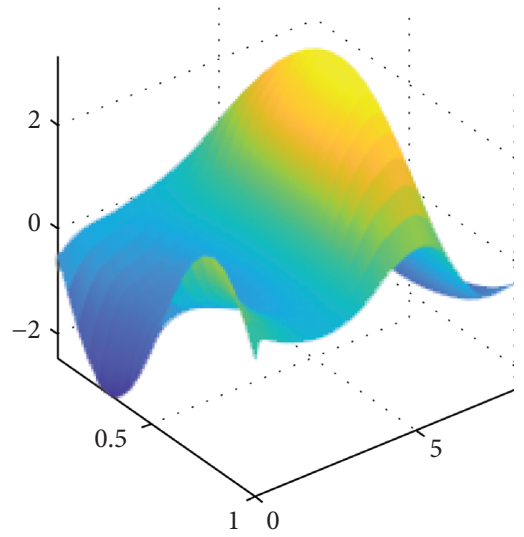

(f)

FIGURE 3: Real part of state error evolution under the output static event-triggered control. net11, net12, and net13 represent 3-dimensional real-valued state evolution of the 1 sterror network. net21, net22, and net 23 represent 3 -dimensional real-valued state evolution of the 2 nd error network. "real" denotes real part. (a) net11-real. (b) net12-real. (c) net13-real. (d) net21-real. (e) net22-real. (f) net23-real. 


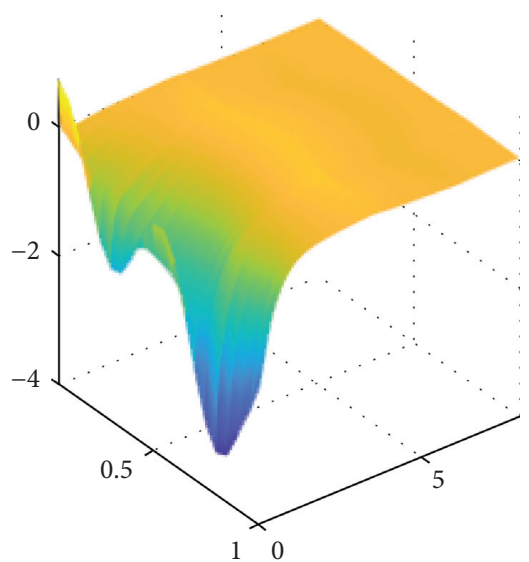

(a)

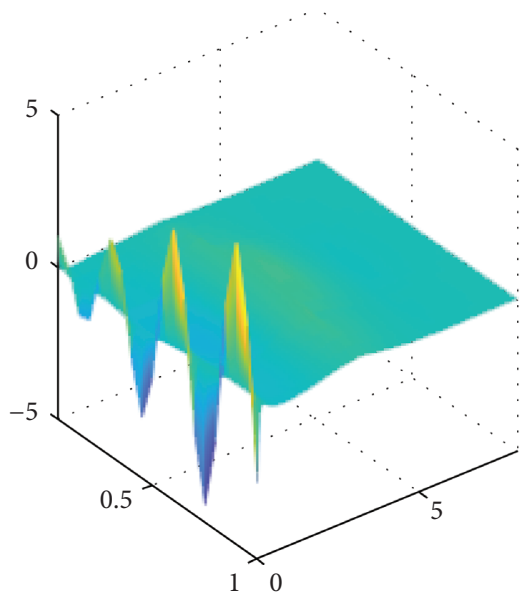

(d)

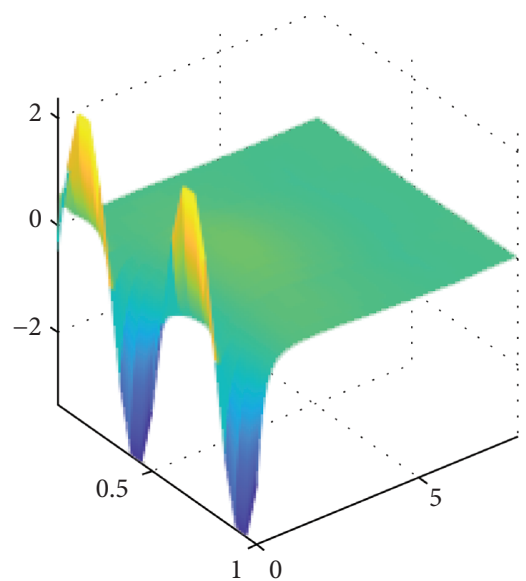

(b)

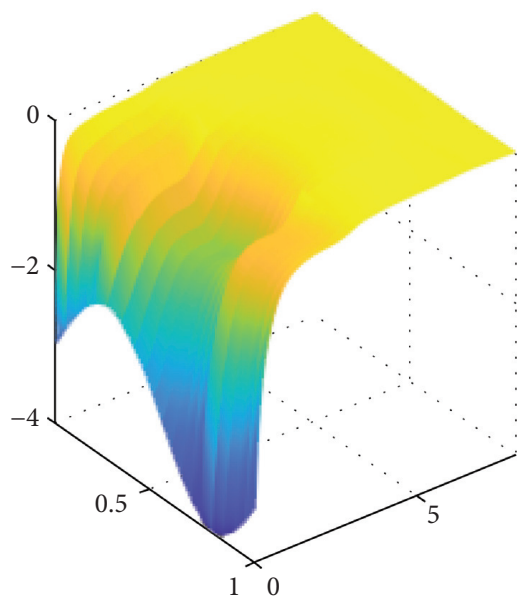

(e)

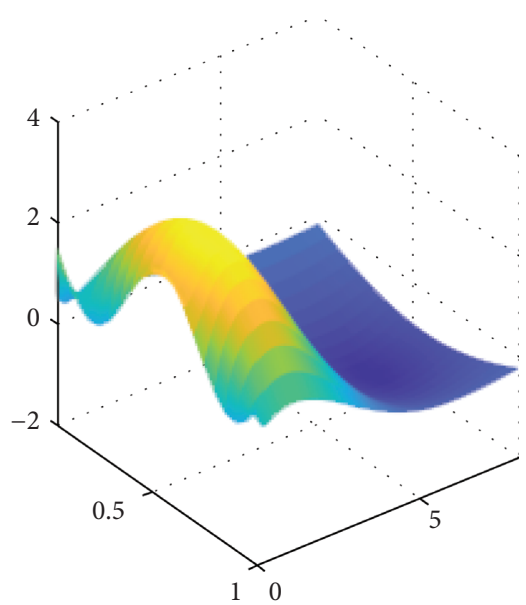

(c)

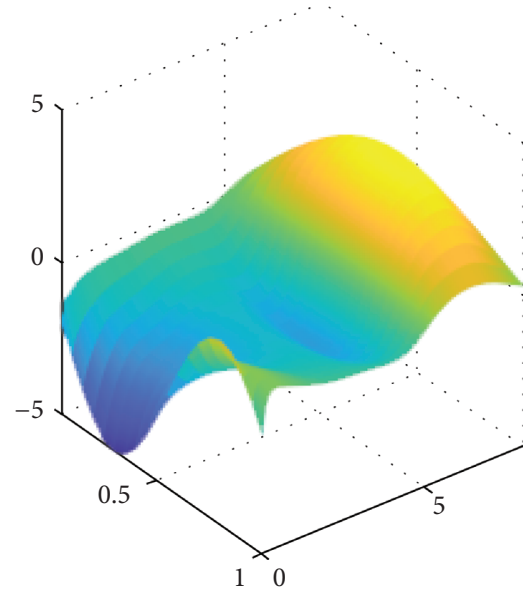

(f)

FIGURE 4: Imaginary part of state error evolution under the output static event-triggered control. net11, net12, and net13 represent 3 dimensional state evolution of the 1 st error network. net21, net22, and net23 represent 3-dimensional state evolution of the 2 nd error network. "im" denotes imaginary part. (a) net11-im. (b) net12-im. (c) net13-im. (d) net21-im. (e) net22-im. (f) net23-im.

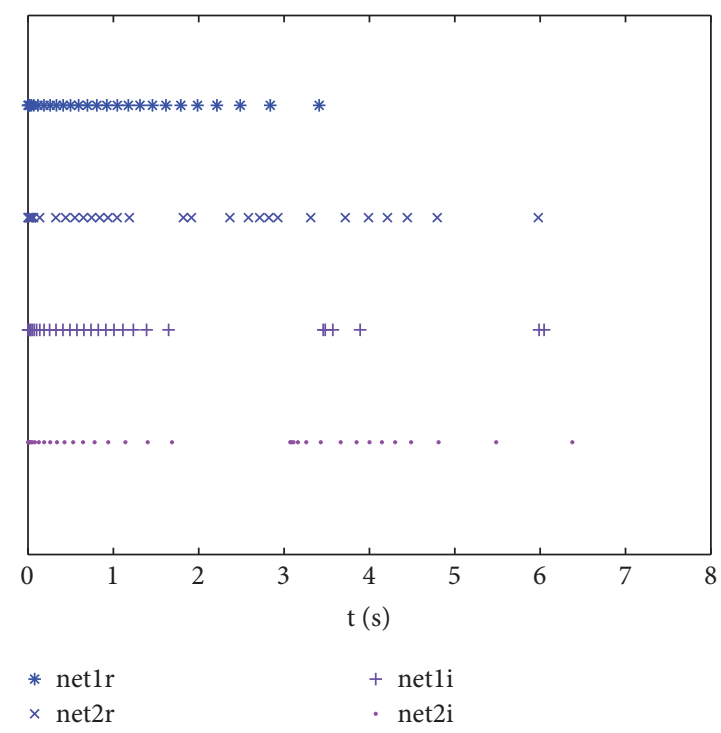

Figure 5: The event-triggered time instants $t_{k}^{i}$ in the error networks (17) under the output static event-triggered control. net1r and net2r mean the real-valued parts of the slave networks 1 and 2. net1i and net2i represent the imaginary parts of the slave networks 1 and 2 , respectively. 


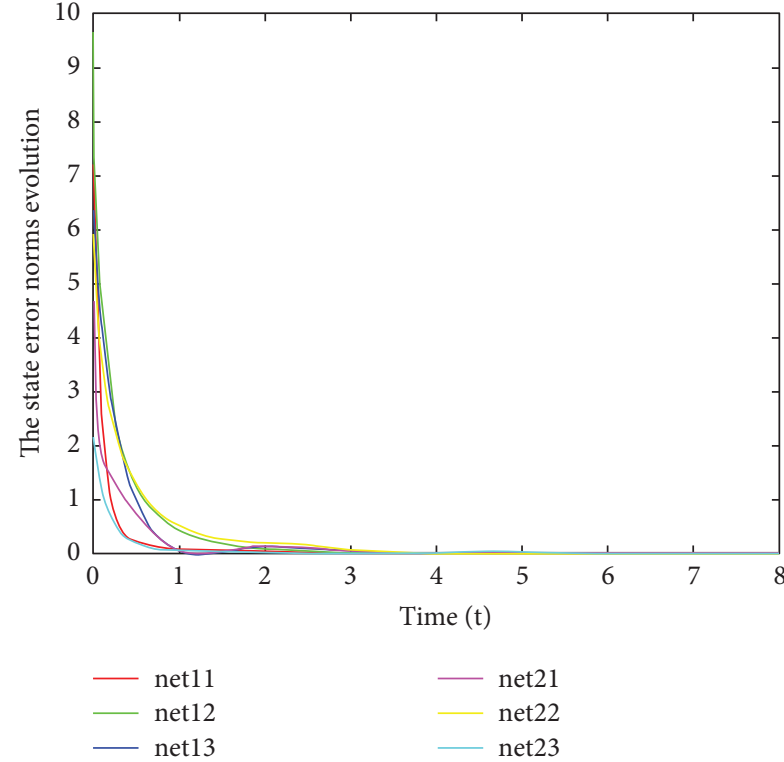

(a)

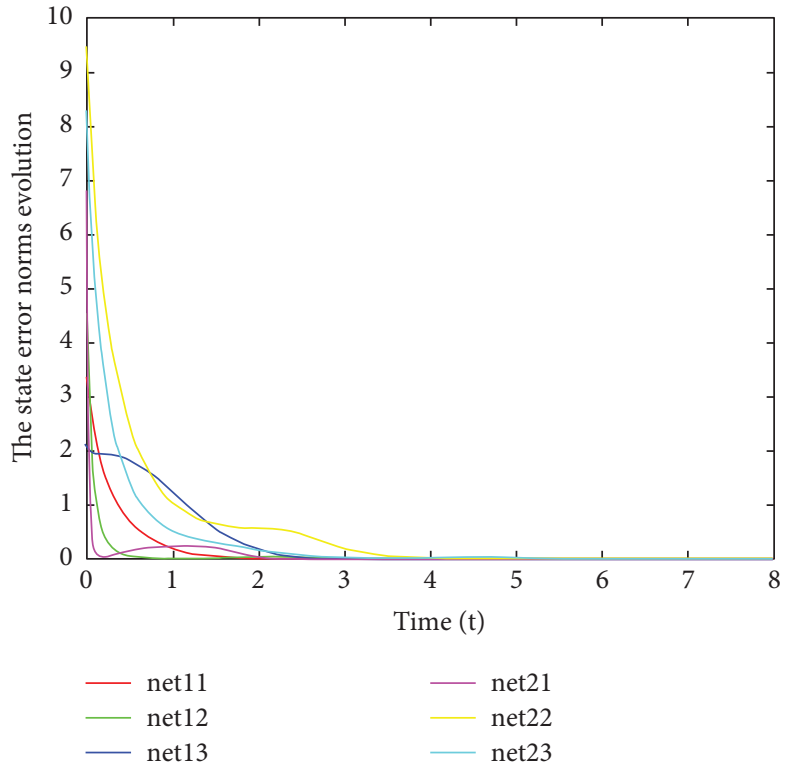

(b)

FIGURE 6: Real part and imaginary part of state error evolution under the piecewise event-triggered control. (a) Real part of state error evolution. (b) Imaginary part of state error evolution.

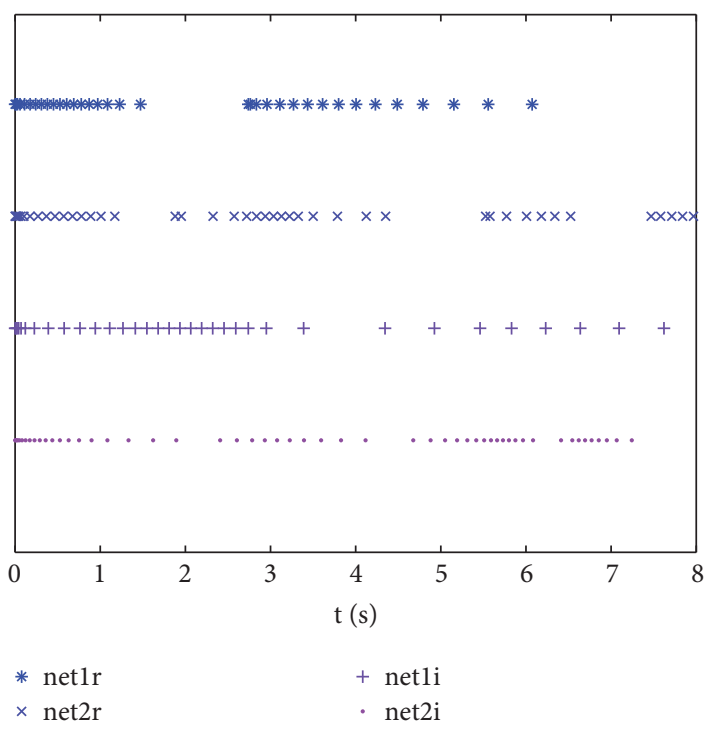

FiguRE 7: The event-triggered time instants $t_{k}^{i}$ in the error networks (17) under the piecewise event-triggered control.

triggered control (OTTC) have been realized where the scale parameter of event trigger is $\gamma_{i}=0.02$; the function parameter of the static event trigger is $\bar{\eta}_{i}(t)=0.01$; the function parameter of the dynamic event trigger is $\bar{\eta}_{i}(t)=0.01 \exp (-0.5 t)$; the output matrix is $\beta=(100 ; 010 ; 001)$; and the time trigger interval is $88 \mathrm{~ms}$. Some results have been achieved and given in Table 1. To compare the update numbers of the controller with different control strategies, the data in Table 1 can be analysed. The data in column 2 and column 3 of Table 1 show that the update rate of OTTC is greater than that of OSETC and ODETC with similar error decreased. The simulation results in columns 4 and 5 imply that the number of controller update increases over time even if the error norm is small enough. Thus, the simulation results demonstrate the first hint; output event-triggered control (OETC) is more effective than output time-triggered control (OTTC), and the similar conclusion can be referred from [36].

The OSETC strategy and ODETC strategy can be further compared using the information in Table 1 . The number of controller update in OSETC shown in column 2 is greater than that of ODETC when state norm is below 0.1. Moreover, the growth rate of controller update in OSETC shown in columns 2 and 4 is lower than that of ODETC. Then, the second hint can be drawn; ODETC is more effective than 
TABLE 1: Six control strategies for synchronization of CMRDNNs.

\begin{tabular}{lcccc}
\hline $\begin{array}{l}\text { Control } \\
\text { strategy }\end{array}$ & $\begin{array}{c}\text { Number of update, state norm } \\
\leq 0.1\end{array}$ & $\begin{array}{c}\text { Time }(\mathrm{s}), \text { state norm } \\
\leq 0.1\end{array}$ & $\begin{array}{c}\text { Number of controller update, } \\
T_{f}=8 \mathrm{~s}\end{array}$ & $\begin{array}{c}\text { State norms } * 10^{3}, \\
T_{f}=8 \mathrm{~s}\end{array}$ \\
\hline OSETC & \multicolumn{2}{c}{ Four values: real part of net1and net2 } & $\begin{array}{c}\text { and imaginary part of net1 and net2 } \\
\text { ODETC }\end{array}$ \\
OTTC & $25,30,26,33$ & $3.5,6.4,4.4,6.4$ & $25,30,28,33$ & $56.4,56.5,6.9,0.7 .4$ \\
& $18,27,20,28$ & $3.4,6.4,4.5,4.9$ & $23,30,25,33$ & $7.0,28.8,18.8,57.4$ \\
\end{tabular}

TABLE 2: Three control strategies for synchronization of CMRDNNs.

\begin{tabular}{lcc}
\hline Control strategy & $T_{f}=8 \mathrm{~s}$, controller update numbers & $T_{f}=15 \mathrm{~s}$, controller update numbers \\
\hline OSETC & $29,32,29,31$ & $31,37,34,40$ \\
ODETC & $26,30,24,31$ & $36,54,34,56$ \\
OPETC & $26,30,24,31$ & $28,31,29,36$ \\
\hline
\end{tabular}

OSETC at the first stage, and OSETC is better than ODETC at the following stage.

Based the above analysis, the distributed output piecewise event-triggered control (OPETC) which combines OSETC and ODETC has been realized, and the results are given in Table 2. Comparing the data in column 2 and column 3 of Table 2, we can find that OPETC has better performance than ODETC and OSETC. This simulation validates the similar theoretical analysis of Theorem 1 .

\section{Conclusions}

The research model in this study is complex and general, including complex-valued states, memristive parameters, state coupling, reaction-diffusion term, and output matrix. First, by combining differential inclusion and nonfragile techniques, a general method is developed to overcome the difficulty of the master-slave system parameter mismatch caused by memristor. Second, an efficient distributed piecewise event-triggered control strategy using incomplete measurement has been designed to achieve output synchronization which not only reduces the update rate of the controller but also decreases the measurement cost. Finally, it is verified that the new distributed piecewise event trigger can achieve better quasisynchronous performance through theoretical analysis and experimental simulation. What is more, the distributed output piecewise event-triggered control strategy can be used for the synchronization control of general one-order reaction-diffusion neural networks without adding any other conditions. To verify wide effectiveness of this control strategy, the OPETC strategy will be extended to two-order or even higher-order neural networks, which is one of our future works.

\section{Data Availability}

The data used to support the findings of this study are available from the corresponding author upon request.

\section{Conflicts of Interest}

The authors declare that there are no conflicts of interest.

\section{Acknowledgments}

This work was supported by the Natural Foundations of China (61773182) and Wuxi Internet of Things Industry funding project.

\section{References}

[1] X. Jin, X. Yu, T. Su, D. Yang, and L. Wang, "Distributed deep fusion predictor for a multi-sensor system based on causality entropy," Entropy, vol. 23, no. 2, pp. 1-19, 2021.

[2] X. Jin, J. Zhang, T. Su, Y. Bai, J. Kong, and X. Wang, "Wavelet-deep optimized model for nonlinear multi-component data forecasting," Computational Intelligence and Neuroscience, 2021.

[3] X.-B. Jin, H.-X. Wang, X.-Y. Wang, Y.-T. au, T.-L. Su, and J.-L. Kong, "Deep-learning prediction model with serial twolevel decomposition based on Bayesian optimization," Complexity, vol. 2020, Article ID 4346803, 14 pages, 2020.

[4] Y. K. Bai and R. Xu, "New synchronization criteria for an array of neural networks with hybrid coupling and timevarying delays," Nonlinear Analysis: Modelling and Control, vol. 21, no. 1, pp. 57-76, 2016.

[5] Y. Luo and Y. Yao, "Finite-time synchronization of uncertain complex dynamic networks with time-varying delay," Advances in Difference Equations, vol. 32, no. 1, pp. 1-22, 2020.

[6] Y. Wang, J. Lu, J. Liang, J. Cao, and M. Perc, "Pinning synchronization of nonlinear coupled Lur'e networks under hybrid impulses," IEEE Transactions on Circuits and Systems II: Express Briefs, vol. 66, no. 3, pp. 432-436, 2019.

[7] D. Ding, Z. Tang, Y. Wang, and Z. Ji, “Adaptive synchronization of complex dynamical networks via distributed pinning impulsive control," Neural Processing Letters, vol. 52, no. 3, pp. 2669-2686, 2020.

[8] L. O. Chua, M. Hasler, G. S. Moschytz, and J. Neirynck, "Autonomous cellular neural networks: a unified paradigm for pattern formation and active wave propagation," IEEE Transactions on Circuits and Systems I: Fundamental Theory and Applications, vol. 42, no. 10, pp. 559-577, 1995.

[9] P.-C. Wei, J.-L. Wang, Y.-L. Huang, B.-B. Xu, and S.-Y. Ren, "Impulsive control for the synchronization of coupled neural networks with reaction-diffusion terms," Neurocomputing, vol. 207, pp. 539-547, 2016.

[10] L. Shanmugam, P. Mani, R. Rajan, and Y. H. Joo, "Adaptive synchronization of reaction-diffusion neural networks and its 
application to secure communication," IEEE Transactions on Cybernetics, vol. 50, no. 3, pp. 911-922, 2018.

[11] L. Wang and J.-L. Wang, "Adaptive passivity and synchronization of coupled reaction-diffusion neural networks with multiple state couplings or spatial diffusion couplings," Neurocomputing, vol. 377, pp. 168-181, 2020.

[12] L. Wang, Y. Jing, and Z. Kong, "Output synchronization of complex network," Journal of Northeastern University. Natural Science, vol. 29, no. 6, pp. 60041-60049, 2008.

[13] J.-L. Wang, H.-N. Wu, T. Huang, and M. Xu, "Output synchronization in coupled neural networks with and without external disturbances," IEEE Transactions on Control of Network Systems, vol. 5, no. 4, pp. 2049-2061, 2018.

[14] D.-Y. Wang, J.-L. Wang, S.-Y. Ren, and Y.-L. Huang, "Output synchronization and Ho output synchronization of complex dynamical networks with multiple derivative couplings," Journal of the Franklin Institute, vol. 356, no. 1, pp. 407-440, 2019.

[15] Q. Wang and J.-L. Wang, "Finite-time output synchronization of undirected and directed coupled neural networks with output coupling," IEEE Transactions on Neural Networks and Learning Systems, vol. 99, pp. 1-12, 2020.

[16] C. Yang, X. Li, and J. Qiu, "Output synchronization control with input constraint of complex networks with reaction-diffusion terms," Neural Computing and Applications, vol. 30, no. 1, pp. 1-8, 2018.

[17] B. Lu, H. Jiang, C. Hu, and A. Abdurahman, "Spacial sampleddata control for Ho output synchronization of directed coupled reaction-diffusion neural networks with mixed delays," Neural Networks, vol. 123, pp. 429-440, 2020.

[18] L. Chua, "Resistance switching memories are memristors," Applied Physics A, vol. 102, no. 4, pp. 765-783, 2011.

[19] W. Zhang, C. Li, T. Huang, and X. He, "Synchronization of memristor-based coupling recurrent neural networks with time-varying delays and impulses," IEEE Transactions on Neural Networks and Learning Systems, vol. 26, no. 12, pp. 3308-3313, 2015.

[20] L. Wang, H. He, and Z. Zeng, "Global synchronization of fuzzy memristive neural networks with discrete and distributed delays," IEEE Transactions on Fuzzy Systems, vol. 28, no. 9, pp. 2022-2034, 2020.

[21] Z. Tu, N. Ding, L. Li, Y. Feng, L. Zou, and W. Zhang, "Adaptive synchronization of memristive neural networks with time-varying delays and reaction-diffusion term," $A p$ plied Mathematics and Computation, vol. 311, pp. 118-128, 2017.

[22] S. Wang, Z. Guo, S. Wen, and T. Huang, "Global synchronization of coupled delayed memristive reaction-diffusion neural networks," Neural Networks, vol. 123, pp. 362-371, 2019.

[23] Y. Liu, X. Liao, and C. Li, "Exponential lag synchronization of memristive neural networks with reaction diffusion terms via neural activation function control and fuzzy model," Asian Journal of Control, vol. 22, no. 1, pp. 346-361, 2018.

[24] S. Wang, Z. Guo, S. Wen, T. Huang, and S. Gong, "Finite/ fixed-time synchronization of delayed memristive reactiondiffusion neural networks," Neurocomputing, vol. 375, pp. 1-8, 2020.

[25] W. Li, X. Gao, and R. Li, "Stability and synchronization control of inertial neural networks with mixed delays," Applied Mathematics and Computation, vol. 367, pp. 1-14, 2020.

[26] H. Akira, "Applications of complex-valued neural networks to coherent optical computing using phase-sensitive detection scheme," Information Sciences Applications, vol. 2, no. 2, pp. 103-117, 1994.

[27] T. Titta, "Orthogonality of decision boundaries in complexvalued neural networks," Neural Comput.vol. 16, no. 1, pp. 73-97, 2004.

[28] S. Zhang, Y. Yang, and X. Sui, "The intermittent control synchronization of complex-valued memristive recurrent neural networks with time-delays," Neural Processing Letters, vol. 50, no. 3, pp. 2119-2139, 2019.

[29] H. Li, X. Gao, and R. Li, "Exponential stability and sampleddata synchronization of delayed complex-valued memristive neural networks," Neural Processing Letters, vol. 51, no. 1, pp. 193-209, 2019.

[30] S. Zhu, D. Liu, C. Yang, and J. Fu, "Synchronization of memristive complex-valued neural networks with time delays via pinning control method," IEEE Transactions on Cybernetics, vol. 50, no. 8, pp. 3806-3815, 2019.

[31] X. Song, J. Man, S. Song, Y. Zhang, and Z. Ning, "Finite/fixedtime synchronization for Markovian complex-valued memristive neural networks with reaction-diffusion terms and its application," Neurocomputing, vol. 414, pp. 131-142, 2020.

[32] X. Jin, X. Lian, T. Su, Y. Shi, and B. Miao, "Closed-loop estimation for randomly sampled measurements in target tracking system," Mathematical Problems in Engineering, vol. 2014, no. 2, pp. 1-12, Article ID 315908, 2014.

[33] T. Li, T. Wang, G. Zhang, and S. Fei, "Event-triggered output synchronization in master-slave Lur'e systems with heterogeneous dimensions," Circuits, Systems, and Signal Processing, vol. 36, no. 2, pp. 811-833, 2017.

[34] T. Li, Y. Yu, T. Wang, and S. Fei, "Decentralized adaptive event-triggered synchronization of neutral networks with time-varying delays," Circuits Systems and Signal Processing, vol. 38, pp. 1-17, 2018.

[35] X. Li, W. Zhang, J. Fang, and H. Li, "Event-triggered exponential synchronization for complex-valued memristive neural networks with time-varying delays," IEEE Transactions on Neural Networks and Learning Systems, vol. 99, pp. 2162-2388, 2019.

[36] S. Wang, Y. Cao, T. Huang, Y. Chen, and S. Wen, "Eventtriggered distributed control for synchronization of multiple memristive neural networks under cyber-physical attacks," Information Sciences, vol. 518, pp. 361-375, 2020.

[37] W. Yao, C. Wang, Y. Sun, C. Zhou, and H. Lin, "Synchronization of inertial memristive neural networks with timevarying delays via static or dynamic event-triggered control," Neurocomputing, vol. 404, pp. 367-380, 2020.

[38] Y. Huang, S. Lin, and E. Yang, "Event-triggered passivity of multi-weighted coupled delayed reaction-diffusion memristive neural networks with fixed and switching topologies," Communications in Nonlinear Science \& Numerical Simulation, vol. 89, pp. 1-28, 2020.

[39] C. A. Swanson, "Wirtinger's inequality," SIAM Journal on Mathematical Analysis, vol. 9, no. 3, pp. 484-491, 1978.

[40] A. Selivanov and E. Fridman, "Delayed point control of a reaction-diffusion PDE under discrete-time point measurements," Automatica, vol. 96, pp. 224-233, 2018.

[41] T. Chen and Z. Cheng, "Synchronization of inertial reactiondiffusion complex networks with mixed time delays via spatiotemporal sampling control," International Journal of Modelling, Identification and Control, 2021.

[42] L. Wen, Y. Yu, and W. Wang, "Generalized halanay inequalities for dissipativity of volterra functional differential equations," Journal of Mathematical Analysis and Applications, vol. 347, no. 1, pp. 169-178, 2008. 
[43] A. D. Ioffe, "Single-valued representation of set-valued mappings II; application to differential inclusions," SIAM Journal on Control and Optimization, vol. 21, no. 4, pp. 641-651, 1983.

[44] R. Zhang, D. Zeng, S. Zhong, and Y. Yu, "Event-triggered sampling control for stability and stabilization of memristive neural networks with communication delays," Applied Mathematics and Computation, vol. 310, pp. 57-74, 2017.

[45] X. Hai, G. Ren, Y. Yu, C. Xu, and Y. Zeng, "Pinning synchronization of fractional and impulsive complex networks via event-triggered strategy," Communications in Nonlinear Science \& Numerical Simulation, vol. 82, pp. 1-16, 2020.

[46] L. Baudouin, S. Marx, and S. Tarbouriech, "Event-triggered damping of a linear wave equation," IFAC-PapersOnLine, vol. 52, no. 2, pp. 58-63, 2019. 